\title{
Kinematics and Dynamics of Multiphase Outflows in Simulations of the Star-forming Galactic Interstellar Medium
}

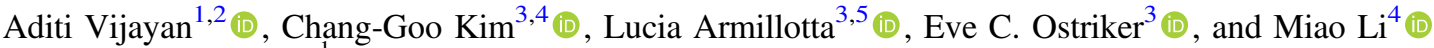 \\ ${ }^{1}$ Raman Research Institute, Bangalore, 500080, India; aditiv@ rri.res.in \\ 2 Joint Astronomy Programme, Department of Physics, Indian Institute of Science, Bangalore 560012, India \\ ${ }^{3}$ Department of Astrophysical Sciences, Princeton University, Princeton, NJ 08544, USA; cgkim@astro.princeton.edu, lucia.armillotta@princeton.edu, \\ eco@astro.princeton.edu \\ ${ }^{4}$ Center for Computational Astrophysics, Flatiron Institute, New York, NY 10010, USA; mli@ flatironinstitute.org \\ ${ }^{5}$ Research School of Astronomy and Astrophysics, The Australian National University, Canberra, ACT, 2611, Australia \\ Received 2019 October 16; revised 2020 March 26; accepted 2020 March 28; published 2020 April 30
}

\begin{abstract}
Galactic outflows produced by stellar feedback are known to be multiphase in nature. Observations and simulations indicate that the material within several kiloparsecs of galactic disk midplanes consists of warm clouds embedded within a hot wind. A theoretical understanding of the outflow phenomenon, including both winds and fountain flows, requires study of the interactions among thermal phases. We develop a method to quantify these interactions via measurements of mass, momentum, and energy flux exchanges using temporally and spatially averaged quantities and conservation laws. We apply this method to a star-forming interstellar medium simulation based on the TIGRESS framework, for solar neighborhood conditions. To evaluate the extent of interactions among the phases, we examine the validity of the "ballistic model," which predicts the trajectories of the warm phase $\left(5050 \mathrm{~K}<T<2 \times 10^{4} \mathrm{~K}\right)$ treated as non-interacting clouds. This model is successful at intermediate vertical velocities $\left(50 \mathrm{~km} \mathrm{~s}^{-1} \lesssim\left|v_{z}\right| \lesssim 100 \mathrm{~km} \mathrm{~s}^{-1}\right)$, but at higher velocities, we observe an excess in simulated warm outflow compared to the ballistic model. This discrepancy cannot be fully accounted for by cooling of highvelocity, intermediate-temperature $\left(2 \times 10^{4} \mathrm{~K}<T<5 \times 10^{5} \mathrm{~K}\right)$ gas. We examine the fluxes of mass, momentum, and energy and conclude that the warm phase gains mass via cooling of the intermediate phase and momentum from the hot $\left(T>5 \times 10^{5} \mathrm{~K}\right)$ phase. The large energy flux from the hot outflow, transferred to the warm and intermediate phases, is quickly radiated away. A simple interaction model implies an effective warm cloud size in the fountain flow of a few $100 \mathrm{pc}$, showing that warm-hot flux exchange mainly involves a few large clouds rather than many small ones.
\end{abstract}

Unified Astronomy Thesaurus concepts: Magnetohydrodynamical simulations (1966); Interstellar medium (847); Galaxy fountains (596); Galaxy winds (626); Stellar feedback (1602)

\section{Introduction}

The formation and evolution of galaxies are regulated by accretion and expulsion of gas. Thus, characterizing the nature and evolution of galactic outflows is of fundamental importance to understanding galaxy evolution. For galaxies that are less massive than the Milky Way, the main mechanism believed to be responsible for ejecting interstellar medium (ISM) is stellar feedback (e.g., Somerville \& Davé 2015; Naab \& Ostriker 2017). Supernova-driven galactic outflows indeed have been directly observed in several nearby star-forming galaxies, revealing a complex multiphase nature: they are composed of hot $\left(T \sim 10^{6}-10^{8}\right.$ K; e.g., Strickland et al. 2004; Strickland \& Heckman 2007), warm ionized $\left(T \sim 10^{5} \mathrm{~K}\right)$, warm neutral ( $T \sim 10^{4}$ K; e.g., Martin 2005; Teng et al. 2013; Chen et al. 2010; Heckman et al. 2015; Chisholm et al. 2017), and cold $\left(T \sim 10^{1}-10^{3} \mathrm{~K}\right)$ molecular (e.g., Walter et al. 2002; Bolatto et al. 2013; Leroy et al. 2015) and atomic gas (e.g., Martini et al. 2018).

In the Milky Way, the presence of gas in extraplanar regions ( $\sim 1-2 \mathrm{kpc}$ above the disk) has been mainly probed by neutral H I (the "Lockman layer"; e.g., Lockman 1984, 2002; Ford et al. 2010; Peek et al. 2011) and ionized $\mathrm{H} \alpha$ emission (the "Reynolds layer"; e.g., Reynolds 1991; Haffner et al. 2003; Gaensler et al. 2008). This extraplanar gas at a temperature of $\sim 10^{4} \mathrm{~K}$ is characterized by disk-like kinematics and metallicities close to the solar value, suggesting a galactic origin (e.g., van Woerden et al. 2004; Marasco \& Fraternali 2011; Qu \& Bregman 2019). Moreover, models aiming to reproduce the extraplanar gas kinematics have shown a negative vertical gradient of the rotational velocity (often called a "lag") and a global infall motion (Marasco \& Fraternali 2011), lending support to a fountain flow origin (e.g., Shapiro \& Field 1976; Bregman 1980). Similar extended warm gas distributions and kinematic signatures are now commonly observed in nearby edge-on galaxies from sensitive $\mathrm{HI}$ observations (e.g., Fraternali et al. 2002; Zschaechner et al. 2011; Marasco et al. 2019) and recent integral field unit surveys of extraplanar $\mathrm{H} \alpha$ (e.g., Bizyaev et al. 2017; Levy et al. 2019).

Cosmological simulations (e.g., Vogelsberger et al. 2014; Schaye et al. 2015; Davé et al. 2016; Pillepich et al. 2018) of galaxy formation focus on the large-scale effects of feedback in the redistribution of gas within and outside galaxies, and the gas flow to and from the circumgalactic medium (CGM). However, large-scale simulations lack sufficient resolution to follow the gas dynamics driven by feedback explicitly. Instead, phenomenological models for feedback (combined with subgrid models of ISM and star formation) are adopted (e.g., Springel \& Hernquist 2003; Vogelsberger et al. 2013) and tuned to yield consistency with, e.g. the stellar-mass-halo-mass relation at redshift zero (e.g., Somerville \& Davé 2015; Naab \& Ostriker 2017, and references therein). To improve the predictive power of large-scale simulations, an important next 
step is to replace phenomenological subgrid wind models with physical models in which the mass (hydrogen and metal), momentum, and energy fluxes in outflows are derived from simulations explicitly treating stellar feedback, the multiphase ISM, and winds, with sufficient resolution.

To this end, outflow properties have been studied in isolated galaxy simulations (e.g., Hopkins et al. 2012; Vijayan et al. 2018; Marinacci et al. 2019; Vasiliev et al. 2019) and cosmological zoom simulations (e.g., Muratov et al. 2015; Christensen et al. 2016; Anglés-Alcázar et al. 2017). However, these simulations do not have high-enough resolution to resolve the evolution of individual supernova remnants (SNRs) or to follow the multiphase interactions involved in launching outflows. Instead, they employ momentum injection schemes for supernova (SN) feedback (e.g., Kimm \& Cen 2014; Hopkins et al. 2018), which do not allow for the direct creation of hot gas from resolved blastwaves (e.g., Kim \& Ostriker 2015a; Rosdahl et al. 2017; Smith et al. 2018; $\mathrm{Hu}$ 2019). A few exceptions exist for simulations of very lowmass dwarf galaxies (Emerick et al. 2019; Hu 2019). With (semi-) Lagrangian or adaptive mesh refinement methods utilized in global simulations, the reduced resolution at low density and/or away from midplane regions implies that the different thermal gas phases in outflows are not separated. As a result, the reported outflow properties in zoom and global galaxy simulations may be based on a numerically mixed, single-phase medium (see Kim \& Ostriker 2018 for a relevant discussion).

In order to understand multiphase outflows and ubiquitous warm fountain flows seen in observations, it is important to analyze simulations that can launch multiphase outflows from first principles and can follow the evolution in the extraplanar region with sufficient resolution to properly capture the interaction between cooler and hotter outflowing phases. Recently, "local patch" simulations of galactic disk regions have begun to incorporate self-consistent star formation (through gravitational collapse) and supernova feedback (e.g., Gatto et al. 2017; Iffrig \& Hennebelle 2017; Kim et al. 2017). One of the chief advantages of local disk simulations is that the uniformly high resolution affords a thorough investigation of multiphase outflows (e.g., Kim \& Ostriker 2018). Wellresolved multiphase outflows, driven by explicitly modeled physical mechanisms, allow us to investigate not only the outgoing mass, momentum, and energy budgets from different phases separately (C.-G. Kim et al. 2020, in preparation; see also Li et al. 2017; Fielding et al. 2018), but also detailed kinematics and dynamics of gas flows and interaction between thermal phases, which is a main focus of this paper.

Physical processes driving the interaction between different gas phases in extraplanar regions have commonly been investigated via small-scale idealized simulations (e.g., Scannapieco \& Brüggen 2015; Schneider \& Robertson 2017; Gronke \& Oh 2018; Sparre et al. 2019, and references therein). These "shock-cloud interaction" simulations model the evolution of warm gas clouds, representing disk material ejected by stellar feedback, which interacts with a more tenuous medium, representing the hotter, higher-velocity outflow phase. These idealized simulations focus on questions such as whether cool clouds can be accelerated without being destroyed, and whether interactions can induce cooling of hot gas. However, a complete characterization of extraplanar gas flows requires analysis of simulations that follow both the origin and evolution of outflowing material, so that spacetime relationships between hot winds and cooler embedded structures will be realistic.

In this paper, we introduce a method to investigate multiphase galactic outflows (wind and fountain flows), testing the conservation and exchange of mass, momentum, and energy between different thermal phases. We apply this analysis to the solar neighborhood model simulated using the TIGRESS (Three-phase ISM in Galaxies Resolving Evolution with Star formation and Supernova feedback) framework for 3D MHD models of the star-forming ISM in galactic disks (Kim et al. 2017). We first characterize the kinematic and dynamical properties of different gas phases. We then compare these properties with the predictions of a simple ballistic model. Finally, we use the conservation laws of hydrodynamics to reveal mass, momentum, and energy flux exchanges between phases. The analysis in this paper is only the first step toward a more complete understanding of multiphase outflow evolution. In the future, this framework can be applied to similar TIGRESS simulations with different galactic conditions (C.G. Kim et al. 2020, in preparation) and potentially to global simulations with sufficiently high resolution (e.g., Schneider et al. 2020). Combining results from studies of this kind, as well as small-scale simulations of cloud-wind interactions and shear layers, an important future goal will be to construct subgrid models of wind launching and evolution that can be applied in cosmological simulations.

This paper is organized as follows: in Section 2, we briefly introduce the TIGRESS framework and discuss the overall extraplanar gas distribution, the time evolution of outflows, and the horizontally and temporally averaged outflow properties in the simulation. In Section 3, we compare the time-averaged velocity probability distribution functions from the simulation with predictions of a model in which individual fluid elements follow ballistic trajectories. In Section 4, we analyze mass, momentum, and energy fluxes to understand how different thermal phases interact with each other during the outflow evolution. We also introduce a simple interaction model to estimate the effective size of clouds. We conclude with a summary of our results and discussion in Section 5.

\section{Solar Neighborhood TIGRESS Model and Its Extraplanar Gas Flows}

\subsection{TIGRESS Framework}

The simulation analyzed in this work was performed using the TIGRESS framework (Kim et al. 2017), in which the starforming ISM is self-consistently modeled. Here we use results from the solar neighborhood model, whose outflow properties are presented in Kim \& Ostriker (2018). In the TIGRESS framework, the ideal magnetohydrodynamics (MHD) equations are solved using the Athena code (Stone et al. 2008; Stone \& Gardiner 2009) in a local box representing a small patch of differentially rotating galactic disk. The solar neighborhood model adopts a galactic rotation rate $\Omega\left(R_{0}\right)=28 \mathrm{~km} \mathrm{~s}^{-1} \mathrm{kpc}^{-1}$ for the center of the domain, with a flat rotation curve $q \equiv$ $-d \ln \Omega / d \ln R=1$ that gives rise to ordered shear of the background azimuthal velocity (local $\hat{\boldsymbol{y}}$ direction) varying along the local radial direction $(\hat{\boldsymbol{x}})$. The horizontal extent of the simulation domain is $-512 \mathrm{pc}<x, y<512 \mathrm{pc}$, with periodic boundary conditions in $y$ and shearing-periodic boundary conditions in $x$ (Stone \& Gardiner 2010). The vertical domain extends to $z= \pm 3584 \mathrm{pc}$ and has outflow boundary conditions. 
The entire numerical domain has a uniform resolution of $4 \mathrm{pc}$. The simulation includes gas self-gravity, optically thin cooling, and spatially uniform grain photoelectric heating by farultraviolet (FUV) radiation. Sink particles are employed to trace the creation of and gas accretion onto star clusters in regions with unresolved collapse. Gravity from and on cluster particles is computed using particle-mesh methods. The stellar feedback from star clusters is modeled in the form of supernova explosions and FUV radiation based on the STARBURST 99 population synthesis model (Leitherer et al. 1999). We refer readers to Kim et al. (2017) for more details.

External gravity from the old stellar disk and the dark matter halo is modeled with a fixed gravitational potential that varies only in the $\hat{z}$ direction (Kuijken \& Gilmore 1989). The functional form used in the simulation and our analysis is

$$
\begin{aligned}
\Phi_{\mathrm{ext}}(z)= & 2 \pi G \Sigma_{*} z_{*}\left[\left(1+\frac{z^{2}}{z_{*}^{2}}\right)^{1 / 2}-1\right] \\
& +2 \pi G \rho_{\mathrm{dm}} R_{0}^{2} \ln \left(1+\frac{z^{2}}{R_{0}^{2}}\right),
\end{aligned}
$$

where $\Sigma_{*}=42 M_{\odot} \mathrm{pc}^{-2}, z_{*}=245 \mathrm{pc}, \rho_{\mathrm{dm}}=0.0064 M_{\odot} \mathrm{pc}^{-3}$, and $R_{0}=8 \mathrm{kpc}$.

\subsection{Extraplanar Gas Distribution}

The simulation begins with a vertically stratified, horizontally uniform gas distribution. The initial conditions assume a doubleexponential density profile, which soon cools (creating a cold phase), with some of the gas collapsing into dense clouds to form star clusters. Young massive stars in newly formed star clusters produce FUV radiation that heats the warm and cold medium, and eventually explode as $\mathrm{SNe}$ that create hot gas and drive turbulence. After the first star formation burst and feedback cycle, the system adjusts its global star formation rates to a self-regulated state in which the feedback maintains the turbulent, thermal, and magnetic support needed to offset the vertical weight of the gas (e.g., Kim et al. 2013; Kim \& Ostriker 2015b; Kim et al. 2017).

While a self-regulated equilibrium state is achieved within the gas disk near the midplane (within the gas scale height, $H \sim 300-400 \mathrm{pc}$ ), some of the gas heated and accelerated by SN blastwaves breaks out into the extraplanar region $(|z|>(1-2) H)$. The outflowing gas includes not only the hot, shock-heated component, but also the highest-velocity portion of the warm gas accelerated by superbubble expansion (Kim \& Ostriker 2018; see also Kim \& Ostriker 2017). To visualize the multiphase structure of gas in the extraplanar region, in Figure 1 we show $\hat{\boldsymbol{y}}-\hat{z}$ slices of the gas number density overlaid with the velocity field (left), and the temperature (middle). We select a simulation snapshot at $t=440 \mathrm{Myr}$ when there is a strong outflow (see below) and slice through $x=140 \mathrm{pc}$. The multiphase nature of the outflowing gas is clearly visible. Warmer $\left(T \sim 10^{4} \mathrm{~K}\right)$ and denser $\left(n \sim 0.1 \mathrm{~cm}^{-3}\right)$ clouds moving with relatively low velocity (few $10 \mathrm{~s} \mathrm{~km} \mathrm{~s}^{-1}$ ) are surrounded by tenuous $\left(n \lesssim 10^{-3} \mathrm{~cm}^{-3}\right)$, hotter $\left(T>10^{6-7} \mathrm{~K}\right)$ gas with $v_{z}>200 \mathrm{~km} \mathrm{~s}^{-1}$. The intermediate-density and -temperature gas is visible at the interface between warm and hot gas and behind the warm clouds as wakes. The gas distribution at $z>0$ is noticeably different from that at $z<0$. The chaotic nature of the turbulent ISM causes asymmetry in the gas distribution and star formation, which results in unequal injection of mass/momentum/energy by feedback in the two regions.

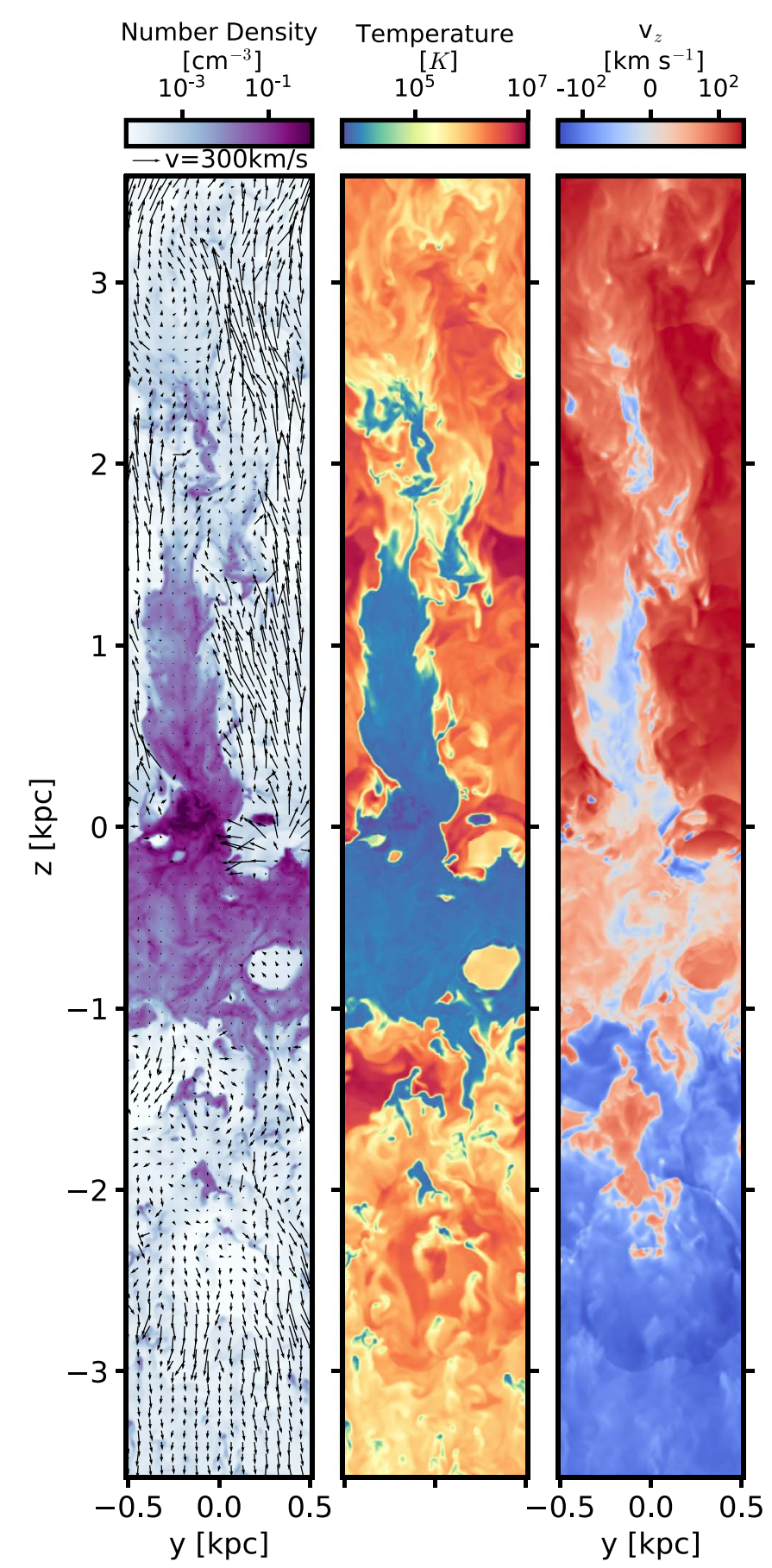

Figure 1. Sample slices at $t=440 \mathrm{Myr}$ through $x=140 \mathrm{pc}$ showing the number density (left panel), temperature (middle panel) and $v_{z}$ (right panel). The arrows overlapping the density slice represent the gas velocity field. Arrow lengths indicate velocity magnitudes. The multiphase nature of the outflowing gas is clearly visible in the temperature slice. Slow-moving warm and dense clouds are surrounded by fast-moving hot and rarefied gas, with intermediatetemperature gas at interfaces and in wakes.

Although star formation is still concentrated near the midplane, $\mathrm{SN}$-driven superbubble evolution and subsequent outflows create large-scale asymmetry in gas density, pressure, and temperature distributions, which affect the net outflow mass, momentum, and energy fluxes above and below the midplane. As a result, our simulation generally shows significant asymmetry between the upper and lower halves of the disk in all quantities presented in this paper. We treat the upper and lower halves separately and present physical quantities separately throughout the paper. 


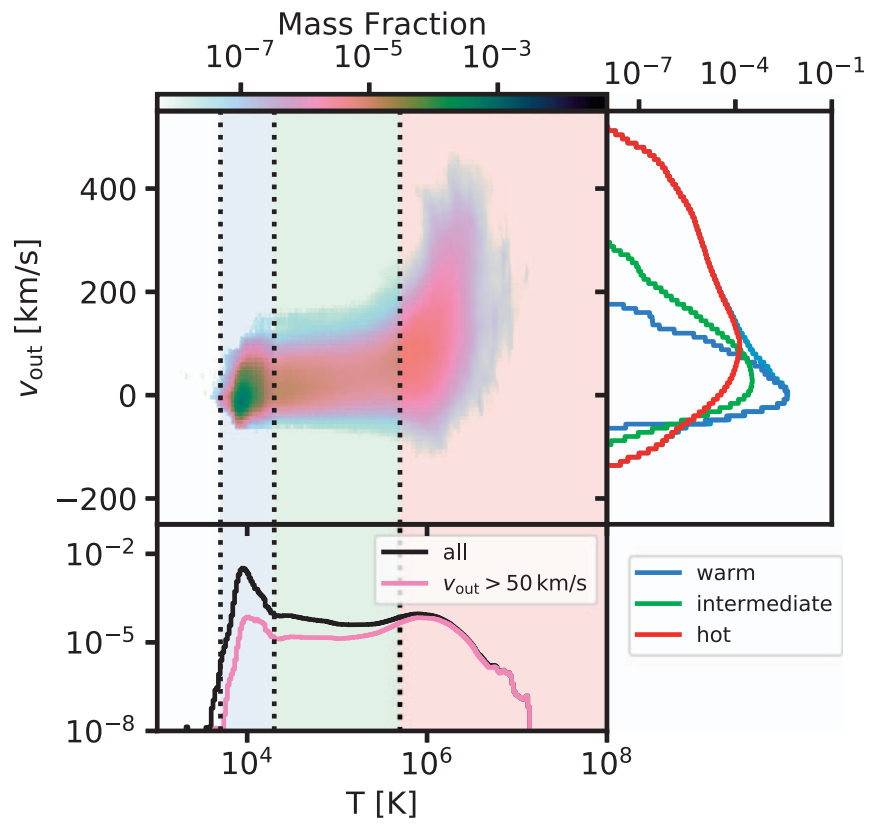

Figure 2. Mass-weighted joint PDF of the extraplanar gas (at $1<z \leqslant 3 \mathrm{kpc}$ ) from the snapshot at $t=440 \mathrm{Myr}$ in the temperature and outflow velocity $\left(v_{\text {out }} \equiv v_{z} \operatorname{sign}(z)\right)$ plane. The blue, green, and red shaded regions in between the vertical dotted lines represent the limits of the corresponding phases. The bottom panel shows the temperature PDF (velocity-integrated, black line; high velocity, pink line). The right panel shows the phase-separated velocity PDFs (key lower right).

For a more quantitative view of the gas distribution, Figure 2 shows a mass-weighted joint probability distribution function (PDF) of all the extraplanar gas $(z>1 \mathrm{kpc})$ for the same time as in Figure 1. The mass within a given temperature and vertical outflow velocity $\left(v_{\text {out }} \equiv v_{z} \operatorname{sign}(z)\right)$ bin is normalized by the total gas mass in the simulation. Note that the extraplanar region comprises only $3 \%$ of the total gas mass. We demarcate the thermal phases using vertical dotted lines and identify three different gas phases in the simulation based on temperature (identical to the definitions adopted in Kim et al. 2017):

1. Warm-5050 K $<T<2 \times 10^{4} \mathrm{~K}$,

2. Intermediate- $2 \times 10^{4} \mathrm{~K}<T<5 \times 10^{5} \mathrm{~K}$,

3. Hot $-5 \times 10^{5} \mathrm{~K}<T$.

Note that cold and unstable phases at $T<5050 \mathrm{~K}$ are negligible in the extraplanar region. We use this nomenclature throughout the paper to refer to gas in different temperature bins.

In the bottom panel of Figure 2, the temperature histogram of all the gas (black curve) shows the dominance of the warm medium by mass $(>90 \%)$. However, if we only consider "high-velocity" gas $\left(v_{\text {out }}>50 \mathrm{~km} \mathrm{~s}^{-1}\right.$, pink curve), the warm and hot components have comparable mass. In the right panel, the phase-separated velocity histograms ${ }^{6}$ show clear development of outflow tails (positive $v_{\text {out }}$ ). We analyze the velocity PDF in greater detail in Section 3.

\subsection{Time Evolution}

We construct horizontally averaged profiles to understand the overall time evolution of gas flows. For each thermal phase, the horizontal average of a quantity $q$ is defined by

\footnotetext{
6 We adopt a consistent color scheme throughout the paper to distinguish the thermal phases: blue for warm, lime green for intermediate, and red for hot.
}

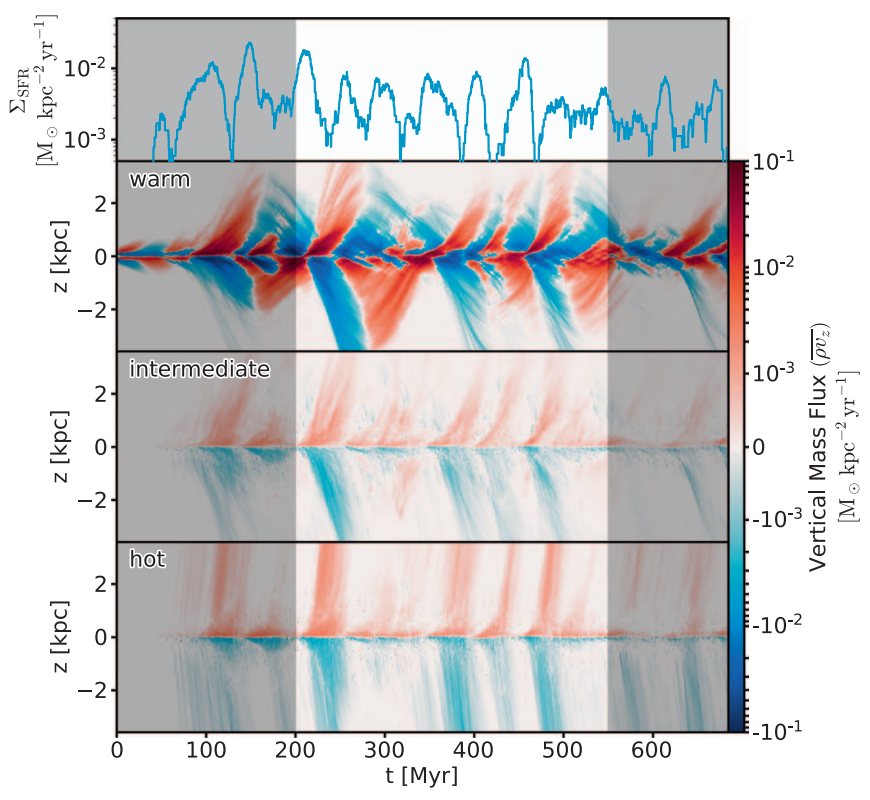

Figure 3. Space-time diagram of horizontally averaged vertical mass fluxes $\overline{\rho v_{z}}$ (see Equation (2)), computed as a function of $z$, for warm, intermediate, and hot phases (bottom three panels). Top panel shows the SFR surface density. Detailed analyses of the simulation are for the central $t=200-550 \mathrm{Myr}$ (gray shaded regions are not included). Star formation is bursty, with the period of $40-50 \mathrm{Myr}$ corresponding to the vertical crossing timescale of the diffuse warm/cold medium. For the portion analyzed, the 16th, 50th, and 84th percentiles of the SFR surface density are $\Sigma_{\mathrm{SFR}}=2.8_{-1.5}^{+4.2} \times 10^{-3} M_{\odot} \mathrm{kpc}^{-2} \mathrm{yr}^{-1}$.

$$
\bar{q}_{\mathrm{ph}}(z ; t)=\frac{\sum_{x, y} q(x, y, z ; t) \Theta_{\mathrm{ph}}(T) \Delta x \Delta y}{L_{x} L_{y}},
$$

where $\Theta_{\mathrm{ph}}(T)$ is the top-hat function that returns 1 for gas at temperatures within the temperature range of each phase (ph = warm, intermediate, or hot) or 0 otherwise, $\Delta x=\Delta y=4 \mathrm{pc}$ is the cell size, and $L_{x}=L_{y}=1024 \mathrm{pc}$ is the horizontal domain size.

Figure 3 shows the spacetime evolution of the horizontally averaged vertical mass fluxes, $\overline{\rho v_{z}}$, for the warm, intermediate, and hot phases. The quantity $\overline{\rho v_{z}}$ represents the mass flux through a horizontal plane; we show the flux at different $z$ values (vertical axis) and different times (horizontal axis). The red/blue color denotes positive/negative mass flux. With this convention, red/ blue corresponds to outflow/inflow for the gas above the midplane, $z>0$; the opposite is true for the lower half of the disk, $z<0$. The time evolution of the warm mass flux at a given height alternates in color, evidence of a fountain in which the majority of the warm outflow falls back because it has been launched with insufficient velocity to escape. In contrast, the color of the hot gas mass flux remains consistent on each side of the midplane. This is because the hot gas has been launched with high-enough velocity to escape the simulation domain as a wind with nearly constant mass flux. However, the intermediatetemperature mass flux decreases as $|z|$ increases (color dilutes), with no clear evidence of inflow following each outflow, implying a transition to the warm phase due to a short cooling time.

In the top panel of Figure 3, we present the time evolution of the SFR surface density measured from the mass of star clusters younger than $10 \mathrm{Myr}$. Star formation is bursty, involving an order of magnitude level fluctuations. However, the system approaches a quasi-equilibrium, self-regulated state, meaning that the average properties do not show a strong secular evolution. We select the 
time range of $t=200-550 \mathrm{Myr}$ for analysis, covering many feedback cycles and outflow events so as to investigate the outflow properties in the statistical steady state. The mean SFR surface density decreases by $20 \%$ within this time range as the system continuously loses gas mass by star formation and outflows.

Within the $t=200-550 \mathrm{Myr}$ time range, we can identify seven to eight star formation peaks. However, from the mass flux evolution, we observe only four clear breakouts (strong mass outflows in all phases). Two starburst events at $t \sim 250-300 \mathrm{Myr}$ do not result in strong outflows. We can see some hints of outflows for these events, but they fail to break out. This is because the large amount of material lifted up after the star formation burst at $t \sim 200 \mathrm{Myr}$ falls back during the subsequent relatively weak star formation events at $t \sim 250$ and $300 \mathrm{Myr}$. On these and other occasions, the conjunction of a "returning" fountain inflow with a subsequent starburst crushes and suppresses what might otherwise be a successful outflow.

\subsection{Time-averaged Properties}

Despite large temporal fluctuations, the overall evolution reaches a quasi-steady state, and we can investigate the timeaveraged quantities to understand the mean behavior. We use simulation outputs between 200 and 550 Myr to construct timeaveraged vertical profiles as

$$
\langle q\rangle_{\mathrm{ph}}(z)=\frac{\sum_{t} \bar{q}_{\mathrm{ph}}(z ; t) \Delta t}{t_{\text {bin }}},
$$

where $\bar{q}_{\mathrm{ph}}(z ; t)$ is the horizontally averaged quantity (Equation (2)), $t_{\text {bin }}=350 \mathrm{Myr}$ is the time range we analyze, and $\Delta t=1 \mathrm{Myr}$ is the time interval of the output dump.

To understand the kinematics and dynamics of outflowing gas, it is crucial to understand the contribution of each component in the momentum equation. ${ }^{7}$ By taking horizontal and temporal averages, explicitly separating and summing over phases, "ph," we obtain the steady-state vertical momentum equation as

$$
\begin{aligned}
& \frac{d}{d z} \sum_{\mathrm{ph}}\left\langle P_{\text {turb }, \mathrm{z}}+P_{\mathrm{th}}+\Pi_{\mathrm{mag}, \mathrm{z}}\right\rangle_{\mathrm{ph}} \\
& =-\sum_{\mathrm{ph}}\left\langle\rho\left(\frac{d \Phi_{\mathrm{ext}}}{d z}+\frac{d \Phi_{\mathrm{sg}}}{d z}\right)\right\rangle_{\mathrm{ph}},
\end{aligned}
$$

where the three terms on the left-hand side (lhs) are the turbulent, thermal, and magnetic force components, respectively, and the two terms on the right-hand side (rhs) are external and self-gravity, respectively. $\Phi_{\text {ext }}$ is the gravitational potential due to the old stellar disk and dark matter halo as prescribed in Equation (1), and $\Phi_{\mathrm{sg}}$ is the gravitational potential of the gas obtained by solving Poisson's equation $\left(\Phi_{\mathrm{sg}}\right.$ includes the negligible contribution from young star clusters). The thermal, $P_{\mathrm{th}}$, and turbulent, $P_{\text {turb,z }}=\rho v_{z}^{2}$, stresses correspond to the thermal and turbulent vertical pressure, ${ }^{8}$

\footnotetext{
7 Note that, for simplicity, here we have dropped the Coriolis force and tidal potential terms arising from the galactic differential rotation included in the simulation. Kim \& Ostriker (2018) presents the full equations, analyzes each term, and concludes that these terms have negligible impact on the outflows we are analyzing in this paper. Note also that on the rhs of Equation (14) in Kim \& Ostriker (2018), $\Phi_{\text {tot }} \nabla \cdot(\rho v)$ is missing.

8 We note that we use the term "turbulent" even though the motions of gas at high altitude are more or less ordered, dominated by either outflow or inflow at a given time that could be considered "ram pressure." However, inflows and outflows do coexist, and the dominance of one or the other alternates in time. Therefore, in a horizontally and temporally averaged sense, the pressure arises from a variance of the vertical velocity, and we simply adopt the nomenclature "turbulent pressure" for any Reynolds stress terms.
}

respectively. However, the magnetic stress, $\Pi_{\mathrm{mag}, \mathrm{z}}=\left(B_{x}^{2}+\right.$ $\left.B_{y}^{2}-B_{z}^{2}\right) /(8 \pi)$, is smaller than the magnetic pressure, $P_{\mathrm{mag}}=$ $\left(B_{x}^{2}+B_{y}^{2}+B_{z}^{2}\right) /(8 \pi)$, due to the magnetic tension term, $-B_{z}^{2} /(4 \pi)$.

By integrating Equation (4) from either the top $\left(z=L_{z} / 2=\right.$ $3584 \mathrm{pc})$ or bottom $\left(z=-L_{z} / 2\right)$ of the simulation domain to the height $z$, we can rewrite the equation in terms of the momentum flux difference (or vertical "support") and the weight of gas:

$$
\sum_{\mathrm{ph}}\left[\mathcal{F}_{p, \mathrm{ph}}(z)-\mathcal{F}_{p, \mathrm{ph}}\left( \pm L_{z} / 2\right)\right]=\sum_{\mathrm{ph}} \mathcal{W}_{\mathrm{ph}}(z) .
$$

Here, the total flux of momentum (subscript "p") in a given phase at $z$ is defined by

$$
\mathcal{F}_{p, \mathrm{ph}}(z) \equiv\left\langle P_{\text {turb }, \mathrm{z}}+P_{\mathrm{th}}+\Pi_{\mathrm{mag}, \mathrm{z}}\right\rangle_{\mathrm{ph}}(z),
$$

and the total weight of gas in a given phase above $z$ is defined by

$$
\mathcal{W}_{\mathrm{ph}}(z) \equiv \int_{ \pm L_{z} / 2}^{z}\left\langle\rho\left(\frac{d \Phi_{\mathrm{ext}}}{d z}+\frac{d \Phi_{\mathrm{sg}}}{d z}\right)\right\rangle_{\mathrm{ph}} d z
$$

Figure 4(a) compares the momentum flux difference $\left(\Delta \mathcal{F}_{p}\right.$, dashed; the lhs of Equation (5)) and the weight of the total gas $(\mathcal{W}$, solid; the rhs of Equation (5)). On average, the vertical support equals the weight of the gas, meaning that the vertical dynamical equilibrium holds very well, as shown in previous works (e.g., Kim et al. 2013; Kim \& Ostriker 2015b). This again justifies the quasi-steady-state assumption. We also show that the gas weight is mostly due to the external gravity, especially above the gas scale height.

In panels (b) and (c), we decompose the vertical support and the weight into different thermal phases. Because the warm gas dominates in terms of mass in the simulation, the weight is dominated by the warm medium everywhere, while the vertical support is provided by both warm and hot phases comparably, especially at high $|z|$. By comparing the vertical support and weight for each phase, we note that the warm gas is lacking in support, while the hot gas shows a large excess in support. The support and weight of the intermediate phase are roughly balanced within the phase without significant excess or deficit.

In order to investigate the momentum flux further, we construct averaged profiles of individual components and phases of the vertical stress. Figure 5 shows the horizontally and temporally averaged vertical profiles of the (a) thermal, (b) turbulent, and (c) magnetic stress terms for different thermal phases, obtained using Equation (3). Near the midplane, $|z|<H,{ }^{9}$ Figure 4(b) shows that the majority of support arises from the warm phase, and Figure 5 shows that the thermal, turbulent, and magnetic components of the warm-phase stress are comparable to each other. The thermal pressure of the hot gas is also significant in this region (Figure 5(a)). At high altitude, $|z|>(1-2) H$, the hot gas dominates both thermal and turbulent pressures. The turbulent pressure of the warm phase is also substantial, but drops rapidly as the warm outflow falls back at $|z| \sim 1-2 \mathrm{kpc}$ (see Figure 3). However, as shown in Figure 4(b), the "support" from the hot and warm phases is similar in this region, as the support arises from the momentum flux "difference." This explains why, although the intermediatephase pressures are always negligible in terms of the absolute values, the vertical support is not negligible (note that the

\footnotetext{
9 " $H$ " is the simulated gas scale height, $H \sim 300-400 \mathrm{pc}$.
} 

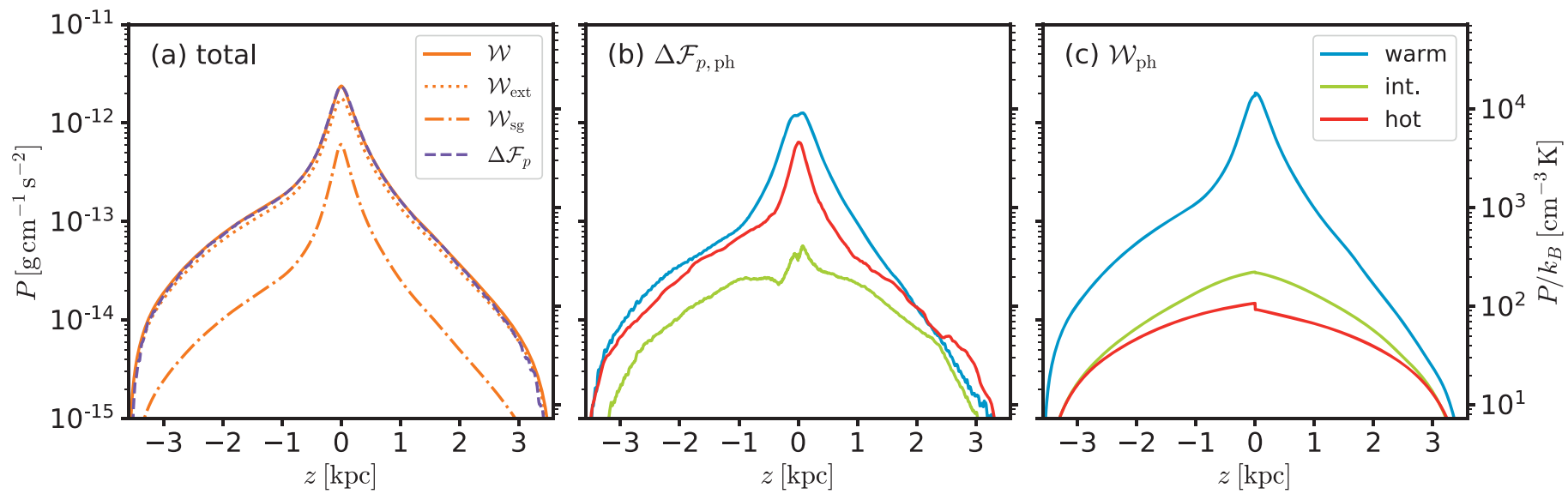

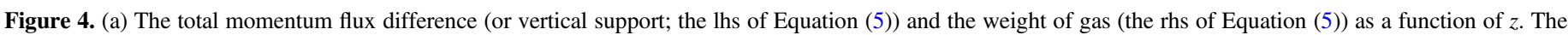

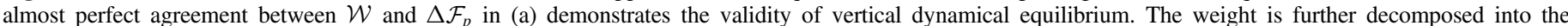

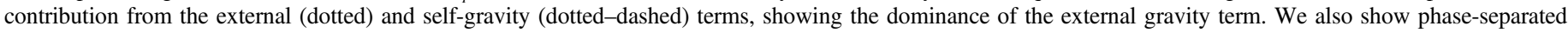
contributions to (b) the momentum flux difference and (c) the weight.
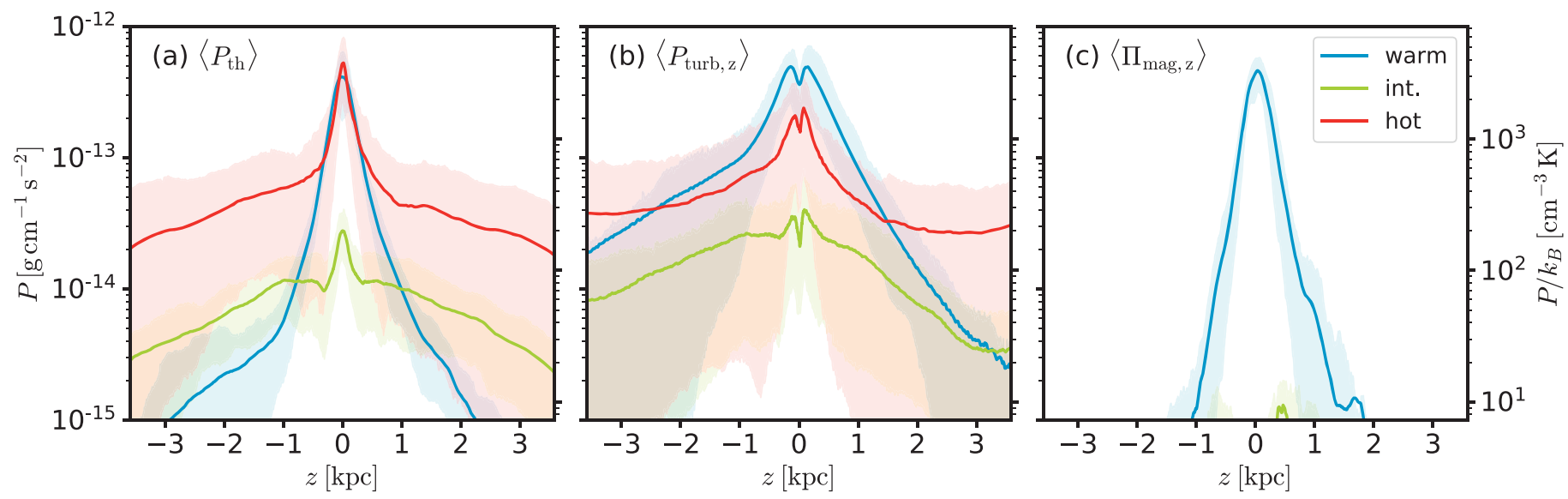

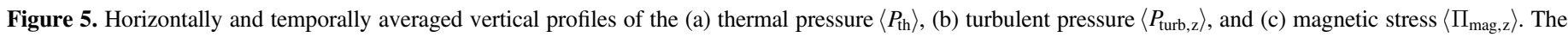
shaded area covers the 16 th and 84 th percentiles of temporal fluctuations.
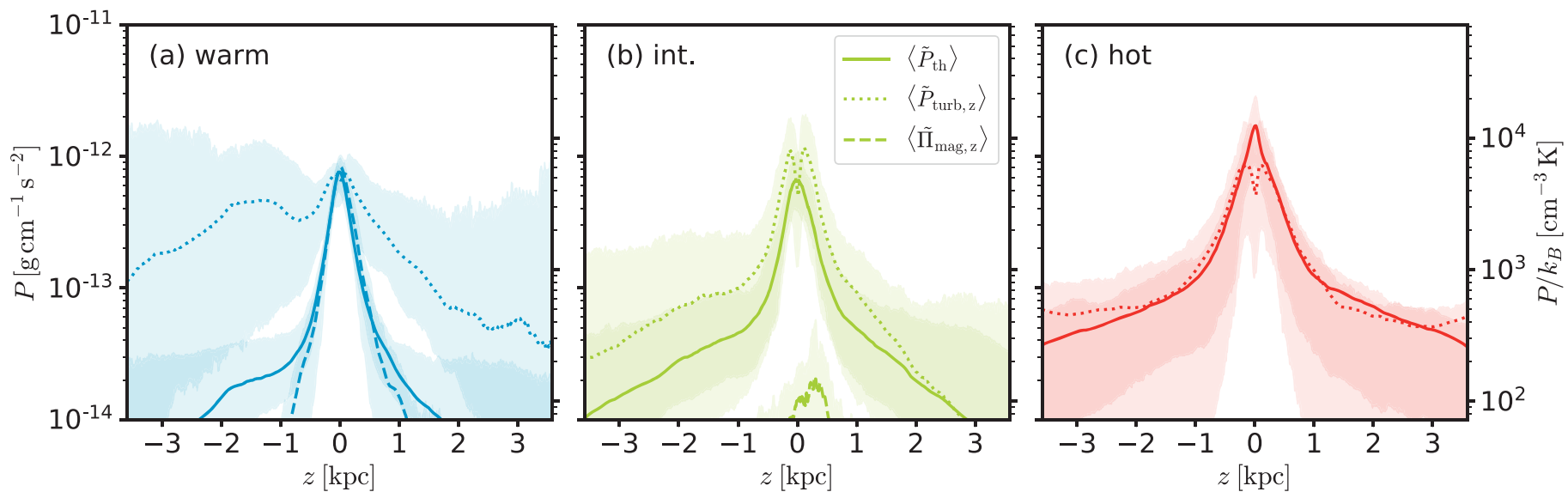

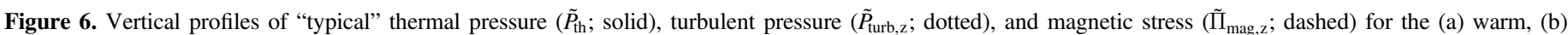

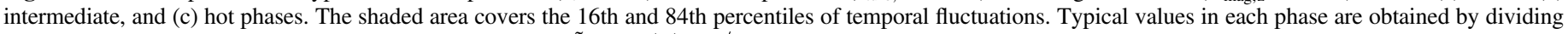
horizontal averages by the area fraction of the phase, e.g., $\tilde{P}_{\mathrm{th}, \mathrm{ph}} \equiv\langle P\rangle_{\mathrm{th}, \mathrm{ph}} / f_{\mathrm{A}, \mathrm{ph}}$.

support of the intermediate phase is mostly compensated by its own weight; see Figures 4(b) and (c)). The magnetic component is negligible in the high-|z| region so that we can safely ignore the magnetic field contribution in the following analysis.
Figure 6 shows the "typical" vertical stress of each component for each phase, obtained by dividing the mean stress by the fraction of area occupied by each gas phase, $f_{\mathrm{A}, \mathrm{ph}} \equiv\langle 1\rangle_{\mathrm{ph}}$, with the definition of the temporal and horizontal averages in Equation (2). 
For example, $\tilde{P}_{\mathrm{th}, \mathrm{ph}} \equiv\left\langle P_{\mathrm{th}, \mathrm{ph}}\right\rangle / f_{\mathrm{A}, \mathrm{ph}}$. In contrast to Figure 5, these vertical profiles show the relative importance of each of the three stress components to the vertical dynamics for a particular phase. Interestingly, the typical turbulent pressure of the warm medium is the largest and dominates the other two at most heights. This figure reinforces the conclusions from Figure 5: (1) for the warm phase, all thermal, turbulent, and magnetic components are comparable near the midplane, while turbulent pressure dominates at high altitude, (2) the hot gas has comparable thermal and turbulent pressures at all heights, and (3) magnetic support is negligible at high altitude.

\section{Ballistic Model of the Warm Outflow}

\subsection{Ballistic Model}

To describe the evolution of the warm outflows $(5050 \mathrm{~K}<$ $\left.T<2 \times 10^{4} \mathrm{~K}\right)$ in the extraplanar region $(1<|z| \leqslant 3 \mathrm{kpc})$, we first consider the simplest model, namely, the ballistic model consistent with the conservation of the mechanical energy: $v_{z}^{2} / 2+\Phi=$ constant. We note that we have taken only the vertical component of the velocity, $v_{z}$, as the change in kinetic energy of the gas in the $x-y$ directions is much smaller.

The ballistic model assumes that each warm gas entity evolves independently, and the change in its velocity is solely due to gravity (no hydrodynamic interactions). Note that external gravity dominates at high altitudes so that $\Phi \approx \Phi_{\text {ext }}$ is a good approximation. Because $\Phi_{\text {ext }}$ is known and fixed in time (see Equation (1)), we can easily calculate the vertical velocity of the warm outflow at an arbitrary height, $z$, from the conditions at launching, $z=z_{i}$, as

$$
v_{z}(z)= \pm \sqrt{v_{i}^{2}-2\left(\Phi(z)-\Phi\left(z_{i}\right)\right)},
$$

where $v_{i}=v_{z}\left(z_{i}\right)$ is the vertical velocity at launching.

As the outflowing gas in the simulation is not launched with a single velocity, it is more informative to consider a velocity PDF (v-PDF). In order to predict the mass-weighted v-PDF, $d M / d v$, at a height $z$, we consider the conservation of mass flux for fluid elements at a given velocity. The mass of gas in a velocity range between $v$ and $v+\delta v$ is given by

$$
\delta M(v)=\frac{d M}{d v} \delta v
$$

while the total mass flux, $\rho v$, can be written as $\delta M(v) v /(A \delta z)$, where $A \delta z$ is a volume of a thin slab that the gas passes through. Assuming mass flux conservation of material in a given velocity range as it travels from $z_{i}$ to $z_{f}$ and that its velocity changes from $v_{i}$ to $v_{f}=v_{z}\left(z_{f}\right)$, we have

$$
\left.\frac{d M}{d v}\right|_{v_{f}} v_{f} \delta v_{f}=\left.\frac{d M}{d v}\right|_{v_{i}} v_{i} \delta v_{i}
$$

From Equation (8), we have $v_{f} \delta v_{f}=v_{i} \delta v_{i}$, so that Equation (10) simply becomes

$$
\left.\frac{d M}{d v}\right|_{v_{f}}=\left.\frac{d M}{d v}\right|_{v_{i}} .
$$

Therefore, under the assumption of mass flux conservation, the $\mathrm{v}$-PDF at $z_{f}$ can be obtained via a velocity shift applied to the initial v-PDF at $z_{i}$ according to the ballistic equation (Equation (8)).

\subsection{Comparison Between Simulation and Model}

Because the typical turbulent pressure of the warm phase is the largest among all the momentum flux terms and phases (see Figure 6), the "zeroth order" expectation is for the warm outflow to evolve more or less ballistically, unaffected by selfinteractions ${ }^{10}$ or interactions with other phases. Therefore, the warm phase is the most suitable component to be compared with the ballistic model.

We compare the mass-weighted v-PDFs of the warm phase as a function of height obtained from the simulation with those predicted by the ballistic model (Section 3.1). For simulation snapshots, we first calculate the mass-weighted v-PDF at each height $z$ and take time averages. For the ballistic model, we use the v-PDF at $\left|z_{i}\right|=1 \mathrm{kpc}$ from the simulation as an initial (launching) condition and calculate the predicted v-PDF at heights $\left|z_{f}\right|=1.5,2,2.5$, and $3 \mathrm{kpc}$ based on Equations (8) and (11). Note that we treat the upper $(z>0)$ and lower $(z<0)$ sides of the simulation domain independently. Assuming a steady injection of outflowing gas through the $z=z_{i}$ plane, both outflowing and inflowing components of the v-PDF at different heights can be predicted (see \pm signs in Equation (8)). Due to the limited vertical extent of the simulation domain, however, outflowing gas with sufficiently high speed is likely to exit the simulation box. Thus, for a fair comparison with the simulation, we set a cutoff velocity in the computation of the inflowing component,

$$
\left|v_{\text {cut }}\right| \equiv \sqrt{2\left[\Phi\left(z_{i}\right)-\Phi\left( \pm L_{z} / 2\right)\right]}=98 \mathrm{~km} \mathrm{~s}^{-1},
$$

where $L_{z}$ is the vertical size of the simulation box. The cutoff velocity determines the maximum infalling speed of the gas.

Figure 7 shows the comparison between simulation and model for the warm phase at heights above (blue) and below (red) the galactic plane, respectively. The quantitative comparison between the simulation data (solid) and the ballistic predictions (dashed) is presented as the fractional difference in the respective bottom panels. The fractional difference is defined by

$$
\Delta \equiv \frac{d M / d v(\text { simulation })-d M / d v(\text { model })}{d M / d v(\text { simulation })} .
$$

A positive or negative $\Delta$ means that the ballistic model underor overpredicts the mass in a velocity bin, respectively.

Despite the highly simplistic assumptions applied here, the ballistic model generally recovers the v-PDF at different heights quite reasonably. In order to construct the v-PDF from the ballistic model, we also ignore interaction between outflow and inflow for a given phase, which certainly exists due to the bursty nature of the star formation and outflows (see Figure 3). Therefore, the overall agreement of the ballistic prediction to the simulation data gets worse as the distance between the heights of launching and prediction gets larger and at higher outflow velocity bins.

The ballistic model underestimates the mass of the warm phase at high-velocity bins (positive $\Delta$ in Figure 7), without overestimating at low-velocity bins (no significant negative $\Delta$ in Figure 7). This potentially means that the high-velocity excess of the warm medium is not due to the acceleration of the low-velocity

\footnotetext{
${ }^{10}$ With self-interactions, collisions of fluid elements with different velocities would yield a fluid element at intermediate velocity. To the extent that the volume filling factor is sufficiently low (see Section 4.2), self-interactions can be ignored.
} 


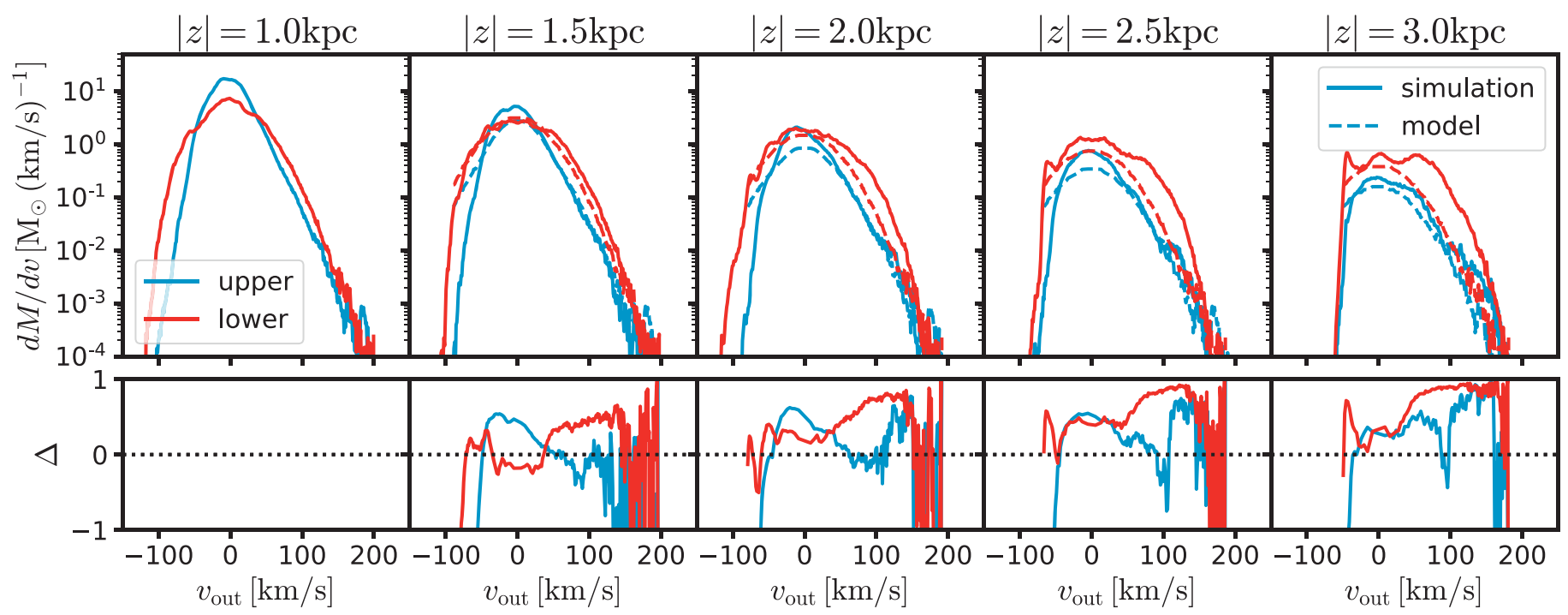

Figure 7. The mass-weighted velocity PDFs for the warm phase along with the fractional difference between the simulation data and the ballistic model, $\Delta=$ (Simulation - Model)/Simulation in the bottom. From left to right, we show the v-PDFs from the simulation data at $|z|=1,1.5,2,2.5$, and $3 \mathrm{kpc}$ as solid lines for the upper $(z>0$; blue) and lower $(z<0$; red) half of the disk. Note that the vertical velocity is multiplied by the sign of $z$ to convert it to the outflow velocity. For $1<|z| \leqslant 3 \mathrm{kpc}$, we plot the ballistic model prediction (see Section 3.1) as the dashed line adopting the v-PDF at $|z|=1 \mathrm{kpc}$ as an initial condition.

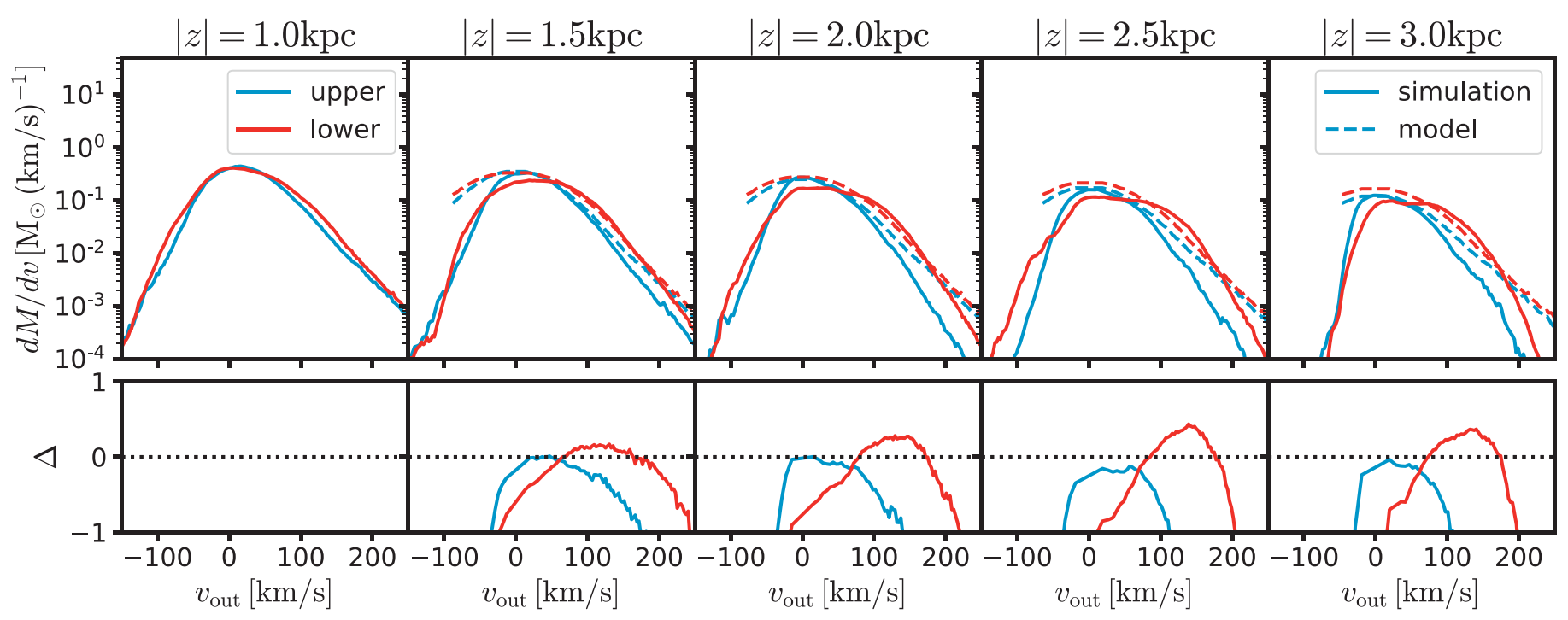

Figure 8. Same as Figure 7, but for the intermediate phase.

warm outflow itself but due to the addition of high-velocity gas from the other phases. Considering the short cooling time of the intermediate phase, it is natural to expect a transition from the intermediate phase to the warm phase. For the intermediate phase, the typical cooling time in $1<|z| \leqslant 3 \mathrm{kpc}$ is $t_{\text {cool }} \equiv\left\langle P_{\mathrm{th}}\right\rangle /$ $[(\gamma-1)\langle\mathcal{L}\rangle] \sim$ few Myr (at $z=1-2 \mathrm{kpc}$ ), where $\mathcal{L}$ is the net volumetric cooling rate, increasing from low altitude to high altitude. This is shorter than or comparable to the outflow crossing time for the simulation domain from $|z|=1 \mathrm{kpc}$ to $|z|=L_{z} / 2=3.6 \mathrm{kpc}, t_{\text {cross }}=\left(L_{z} / 2-1 \mathrm{kpc}\right) / v_{\text {out }}=25 \mathrm{Myr}$ $\left(v_{\text {out }} / 100 \mathrm{~km} \mathrm{~s}^{-1}\right)^{-1}$.

To further test the idea that cooling of intermediatetemperature gas $\left(2 \times 10^{4} \mathrm{~K}<T<5 \times 10^{4} \mathrm{~K}\right)$ produces high-velocity warm gas at large $z$, we perform the same comparison for the intermediate phase in Figure 8. Here, we do not anticipate the general validity of the ballistic model for the intermediate phase as the non-negligible cooling in the intermediate phase violates the necessary assumptions for the ballistic model (mass conservation). Note that, although the phase transition due to cooling of the intermediate phase also means the addition of mass to the warm phase, the mass contribution from the intermediate phase is not dominant for the warm phase so that the ballistic assumption we have made for the warm phase may still hold approximately (see Section 4.1 for details). Bearing these caveats in mind, Figure 8 shows general deficits of the mass at high-velocity bins of intermediate-temperature gas (negative $\Delta$ ), without significant excesses in any other velocity bins. Because the mass fraction at high-velocity bins is smaller, the excess would be more prominent at these bins.

If we simply assume that the gas at intermediate temperature cools and turns into the warm phase, the phase transition just moves the mass at a given velocity bin from one phase to the other. As shown in Figure 9, when we consider the warm and intermediate phases together, the agreement is only partly improved. In particular, the excess of the warm phase on the 


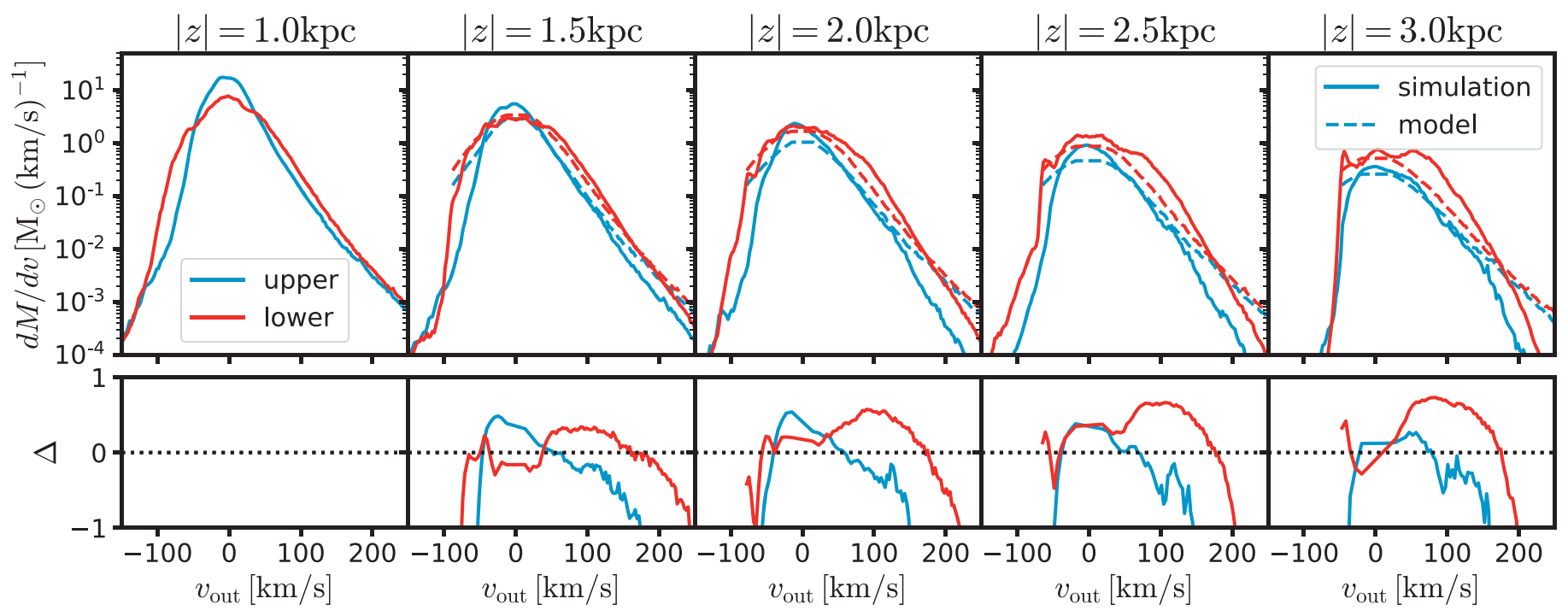

Figure 9. Same as Figure 7, but for the sum of the warm and intermediate phases.

upper side of disk at $50 \mathrm{~km} \mathrm{~s}^{-1}<v_{\text {out }}<150 \mathrm{~km} \mathrm{~s}^{-1}$ is well counterbalanced by the deficit of the intermediate phase. However, on the lower side of disk, the intermediate phase does not make the ballistic model better.

So far, we have ignored any dynamical interaction between phases. However, the v-PDFs shown in Figures 7-9 possess a signature of phase interaction. The outflow is generally asymmetric (see Figures 1 and 3 ), and this is evident in Figure 7, where the v-PDFs of the warm phase at $z= \pm 1 \mathrm{kpc}$ show a significant difference. In contrast, the intermediate phase shows very similar v-PDFs at $|z|=1 \mathrm{kpc}$ for both sides. The asymmetry in the intermediate phase emerges as it moves outward and interacts with different warm-phase outflows. In addition to the failure of the simple cooling idea, this clearly implies that the dynamical interaction between phases exists and affects the evolution of outflows noticeably. We quantify this effect in the next section.

\section{Mass, Momentum, and Energy Transfers between Phases}

In order to provide a more complete picture of multiphase outflows and interaction between phases, we now analyze key terms in the hydrodynamic equations of mass, momentum (in the $z$ direction), and energy conservation (we ignore magnetic fields in this section as we already saw that the magnetic terms are negligible in outflows; see Section 2.4). We first take the horizontal average for each thermal phase and the temporal average for the time range of $t \in\left(t_{1}, t_{2}\right)$ as defined in Equations (2) and (3). Then, we further integrate the equations outward along the vertical direction from $z_{i}$ to $z$, where $z_{i}= \pm 1 \mathrm{kpc}$. As we shall show in Section 4.1, we treat the upper and lower halves of the disk separately.

The set of hydrodynamic partial differential equations yields integrated relationships based on the three conservation laws for mass, momentum, and energy:

$$
\begin{gathered}
\sum_{\mathrm{ph}}\left[\langle\dot{M}\rangle_{\mathrm{ph}} / A+\Delta_{z} \mathcal{F}_{M, \mathrm{ph}}\right]=0, \\
\sum_{\mathrm{ph}}\left[\langle\dot{p}\rangle_{\mathrm{ph}} / A+\left(\Delta_{z} \mathcal{F}_{p, \mathrm{ph}}-\Delta_{z} \mathcal{W}_{\mathrm{ph}}\right)\right]=0,
\end{gathered}
$$

$$
\sum_{\mathrm{ph}}\left[\langle\dot{E}\rangle_{\mathrm{ph}} / A+\Delta_{z} \mathcal{F}_{E, \mathrm{ph}}\right]=-\mathcal{L}_{\mathrm{ph}}+\dot{E}_{\mathrm{SN}} / A .
$$

The rate of change in mass within the volume of interest, $\langle\dot{M}\rangle_{\mathrm{ph}}$, is defined by

$$
\langle\dot{M}\rangle_{\mathrm{ph}} \equiv \sum_{z^{\prime}=z_{i}}^{z}\left\langle\frac{\partial \rho\left(z^{\prime}\right)}{\partial t}\right\rangle_{\mathrm{ph}} A \Delta z,
$$

where $\Delta z$ is the cell size and $A=L_{x} L_{y}$ is the total area of the horizontal plane. Similarly, $\langle\dot{p}\rangle_{\mathrm{ph}}$ and $\langle\dot{E}\rangle_{\mathrm{ph}}$ are defined by replacing $\rho$ in Equation (16) with $\rho v_{z}$ and by $\rho v^{2} / 2+$ $P_{\text {th }} /(\gamma-1)$, respectively, where $\gamma=5 / 3$ is the adiabatic index.

The momentum flux $\mathcal{F}_{p \text {,ph }}$ in phase "ph" is defined by Equation (6), while the mass and energy fluxes are, respectively, defined by

$$
\mathcal{F}_{M, \mathrm{ph}} \equiv\left\langle\rho v_{\mathrm{out}}\right\rangle_{\mathrm{ph}}
$$

and

$$
\mathcal{F}_{E, \mathrm{ph}} \equiv\left\langle\rho v_{\mathrm{out}} \mathcal{B}\right\rangle_{\mathrm{ph}},
$$

where the Bernoulli parameter is

$$
\mathcal{B} \equiv \frac{v^{2}}{2}+\frac{\gamma}{\gamma-1} \frac{P_{\text {th }}}{\rho}+\Phi .
$$

The weight of the gas $\mathcal{W}_{\mathrm{ph}}$ is defined by Equation (7). For the mass, momentum, and energy fluxes, and for the weights, $\Delta_{z} q \equiv q(z)-q\left(z_{i}\right)$, i.e., the difference between the value at the height of interest $z$ and the outflow launching point $z_{i}$.

The source terms in Equation (15) indicate the energy loss by cooling $\mathcal{L}_{\mathrm{ph}} \equiv \sum_{z^{\prime}=z_{i}}^{z}\left\langle n^{2} \Lambda(T)-n \Gamma\right\rangle_{\mathrm{ph}}\left(z^{\prime}\right) \Delta z$ and the gain by direct $\mathrm{SN}$ energy injection $\dot{E}_{\mathrm{SN}} \equiv 10^{51} \mathrm{erg} N_{\mathrm{SN}} / t_{\text {bin }}$.

The first term on the lhs of Equations (13)-(15) is the change of mass, momentum, and energy in each thermal phase within the volume and time interval of interest. If the system is in a perfect steady state, the summation over thermal phases would be zero. Due to the dynamic and bursty nature of the simulation, the steady-state assumption within the volume we are analyzing is not always satisfied even after the long 
temporal averaging $\left(t_{1}=200 \mathrm{Myr}\right.$ and $\left.t_{2}=550 \mathrm{Myr}\right)$. We thus keep this term to demonstrate how significant the unsteady behavior is. The total mass difference can be particularly large compared to the mass flux term. Although we consider the volume far from the midplane $(1 \mathrm{kpc}<|z| \leqslant 3 \mathrm{kpc})$, there is still direct $\mathrm{SN}$ energy injection due to $\mathrm{SNe}$ from runaways, which serves as a source term in the energy equation.

\subsection{Simulation Results}

In order to understand mass exchange between phases, we plot individual terms of Equation (13) in Figure 10. ${ }^{11}$ In the absence of interactions between phases, we would expect that the increase (decrease) in mass flux for each phase would be balanced by an equivalent decrease (increase) in $\langle\dot{M}\rangle$ for each phase. In other words, each phase would individually satisfy Equation (13) (the solid and dashed lines in Figure 10 would compensate each other color by color). This is not the case.

For the hot phase (red; $T>5 \times 10^{5} \mathrm{~K}$ ), the mass flux difference and the corresponding $\langle\dot{M}\rangle_{\mathrm{h}}$ are both nearly zero, showing that the hot gas coming in from $z_{i}$ simply goes out without having significant mass exchange interactions with either of the two phases. The intermediate phase (lime green; $2 \times 10^{4} \mathrm{~K}<T<5 \times 10^{5} \mathrm{~K}$ ) has negligible $\langle\dot{M}\rangle_{\mathrm{i}}$, while its mass flux difference (divergence) is substantial and negative. This means that the intermediate-phase gas that comes in from the bottom of the volume neither escapes outward nor stays in the volume to increase the mass of the intermediate- $T$ gas. This points to the fact that mass exchange must exist between the warm (blue; $5050 \mathrm{~K}<T<2 \times 10^{4} \mathrm{~K}$ ) and intermediate phases.

Within the time interval considered, the mass flux difference $\left(\Delta_{z} \mathcal{F}_{M, \mathrm{w}}\right)$ of the warm phase is positive. This could correspond either to net flow outward through the top of the box (into the CGM) or inward through the bottom of the box (a falling fountain) ${ }^{12}$ The total mass change within the period is solely due to the warm phase (the blue dashed line is almost perfectly overlaid by the black dashed line representing all gas) and smaller than the mass flux difference (magnitude of the blue solid line is larger than the blue/black dashed line). More warm gas flows out of the volume than the rate of change of the stored warm mass, necessitating a transfer of mass flux from the intermediate-temperature to the warm gas.

In short, Figure 10 shows three robust features of the mass flux difference: (1) the hot gas mass flux is nearly constant with height, $\Delta_{z} \mathcal{F}_{M, \mathrm{~h}} \sim 0$, (2) the intermediate gas mass flux decreases with height, $\Delta_{z} \mathcal{F}_{M, \mathrm{i}}<0$, without producing an increase in the mass $\langle\dot{M}\rangle_{\mathrm{i}} \sim 0$, and (3) the warm gas mass flux

\footnotetext{
${ }^{11}$ We would like to note that our finding that the warm mass flux increases with $|z|$ might seem in apparent contradiction with what is shown in Figure 7 of Kim \& Ostriker (2018). There, the mass fluxes of the warm gas during the outflow-dominated (Figure 7(a)) and inflow-dominated (Figure 7(b)) periods are analyzed separately, yielding the result that the absolute value of the mass flux decreases with $z$ in both periods. Here, we analyze the mass flux over the entire selected temporal range without making any distinction between outflow-dominated and inflow-dominated periods. Thus, the mass flux here $\left(\mathcal{F}_{M, \mathrm{w}} \equiv\left\langle\rho v_{z}\right\rangle_{\mathrm{w}}\right)$ should be seen as the difference between the positive mass flux during the outflow-dominated period and the negative value during the inflow-dominated period in Kim \& Ostriker (2018).

12 Because warm gas velocities are usually insufficient to escape from the galaxy, a positive divergence in the flux would generally be expected to represent an inward fountain flow. However, the vertical extent of this simulation domain is limited, so that a strong burst can sometimes lead to substantial net outflow for the warm phase. This in fact occurs at $\sim 250 \mathrm{Myr}$ in the lower half (see Figure 3).
}
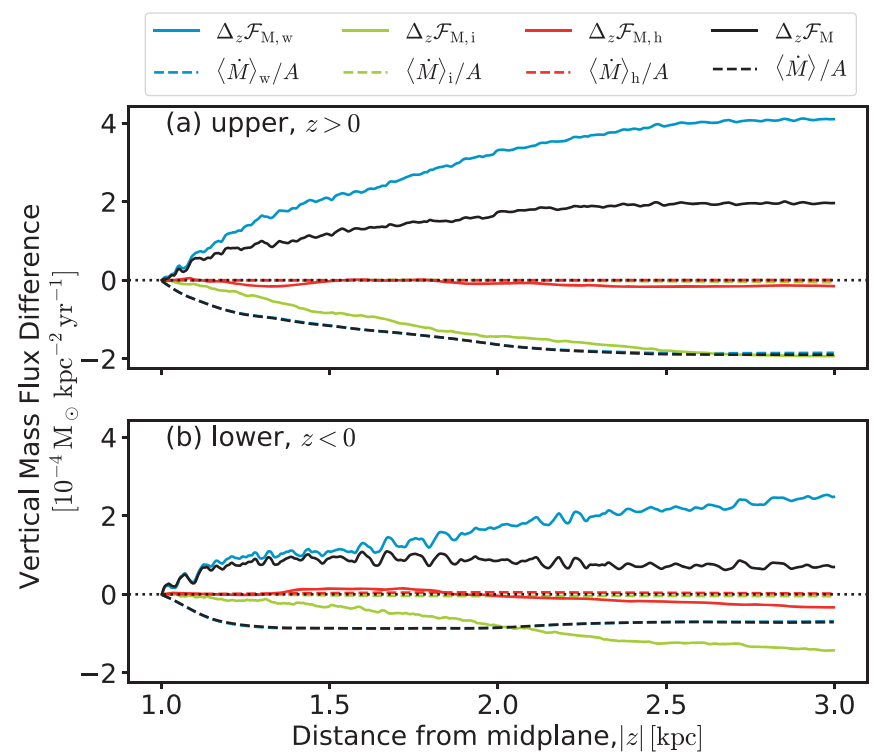

Figure 10. Each term of Equation (13), showing the rate of mass change per area $(\langle\dot{M}\rangle / A$, Equation (16); dashed) and the vertical mass flux difference between $z_{i}=1 \mathrm{kpc}$ and $z\left(\Delta_{z} \mathcal{F}_{\mathrm{M}}\right.$, Equation (17); solid) for each phase (blue for warm, lime green for intermediate, and red for hot) as well as the whole gas (black) in the (a) upper and (b) lower sides of the disk. Note that the mass change over the time period we consider is negligibly small for the intermediate and hot phases and significant for the warm phase, $\langle\dot{M}\rangle \sim\langle\dot{M}\rangle_{\mathrm{w}} \gg\langle\dot{M}\rangle_{\mathrm{i} / \mathrm{h}}$, making the blue dashed line invisible under the dashed black line.

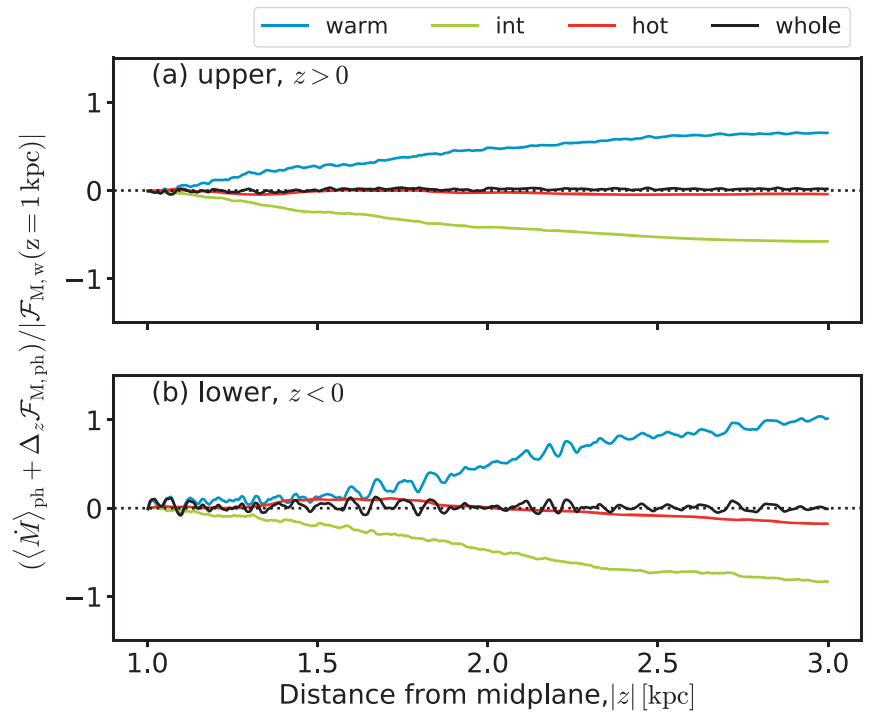

Figure 11. Net mass flux difference in each thermal gas phase $\left(\langle\dot{M}\rangle_{\mathrm{ph}} / A+\Delta_{z} \mathcal{F}_{\mathrm{M}, \mathrm{ph}}\right)$ between the height of interest $z$ and launching $z_{i}=1 \mathrm{kpc}$. Separately shown are the (a) upper and (b) lower sides of the disk. All terms are normalized by the mass flux of the warm phase at $z=z_{i}$. The net mass gained by the warm phase matches the mass flux lost by the intermediate- $T$ phase, $\langle\dot{M}\rangle_{\mathrm{w}} / A+\Delta_{z} \mathcal{F}_{\mathrm{M}, \mathrm{w}} \approx-\Delta_{z} \mathcal{F}_{\mathrm{M}, \mathrm{i}}$.

increases with height, $\Delta_{z} \mathcal{F}_{M, \mathrm{w}}>0$. The increase in warm mass flux with height can be attributed to a combination of flux transfer from the intermediate phase and a reduction in the "stored" warm mass over the period.

To directly compare the mass flux loss and gain within each phase, in Figure 11 we show the net mass flux gain per unit area per unit time of each phase $\langle\dot{M}\rangle_{\mathrm{ph}} / A+\Delta_{z} \mathcal{F}_{M, \mathrm{ph}}$, normalized by the mass flux at $\left|z_{i}\right|=1 \mathrm{kpc}$ of the warm phase $\left|\mathcal{F}_{M, \mathrm{w}}\left(z_{i}\right)\right|$. 


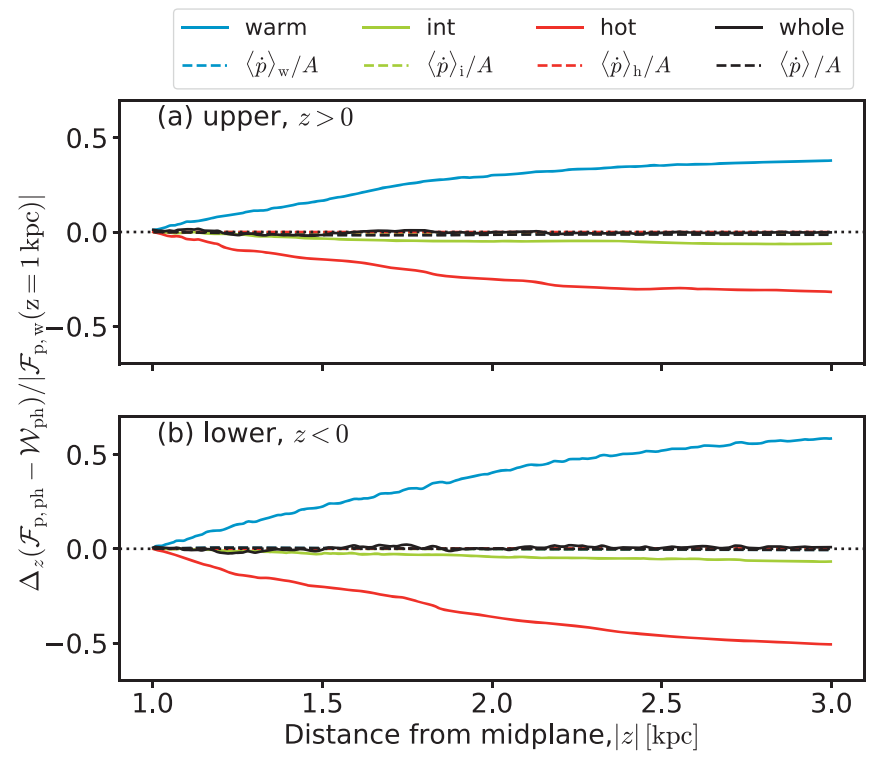

Figure 12. Same as Figure 11, but for the net momentum flux difference $\Delta_{z}\left(\mathcal{F}_{\mathrm{p}, \mathrm{ph}}-\mathcal{W}_{\mathrm{ph}}\right)$ (found using Equations (6) and (7); solid line) taking into account the flux loss due to gas climbing out of the potential; also shown is the momentum change rate per unit area $\langle\dot{p}\rangle_{\mathrm{ph}} / A$ (found by substituting $\rho \rightarrow \rho v_{z}$ into Equation (16); dashed line). Note that the momentum changes are negligibly small for each phase individually as well as for the whole gas. Although the mass change rate is non-negligible for the warm phase (see Figure 10), its momentum change is small due to the preferentially low velocity of the warm phase (see Figure 7).

Over the height range we consider here, the warm phase gains mass flux from the intermediate phase nearly continuously. Between 1 and $3 \mathrm{kpc}$, the decrease in the intermediate- $T$ flux, $\Delta_{z} \mathcal{F}_{M, \mathrm{i}}$, and the corresponding increase in the warm mass flux, amounts to $50 \%-100 \%$ of the launch value of $\mathcal{F}_{M \text {,w }}$ (at $|z|=1 \mathrm{kpc}$ ).

Next, we investigate momentum exchanges between phases. Figure 12 plots the "net" momentum flux difference $\Delta_{z}\left(\mathcal{F}_{p, \mathrm{ph}}-\mathcal{W}_{\mathrm{ph}}\right)$ rather than the momentum flux itself, which always decreases as the outflow climbs up the gravitational potential well, i.e., $\Delta_{z} \mathcal{F}_{p \text {,ph }}<0$ (see Figure 4 ). We also plot $\langle\dot{p}\rangle_{\mathrm{ph}} / A$ to show that these terms are negligible and hence do not contribute significantly to momentum balance among the phases. The total net momentum flux difference (black) is nearly zero because vertical equilibrium holds at every height, as we see in Figure 4. However, significant momentum flux loss occurs in the hot phase, which clearly corresponds to a gain in the warm phase. Although there is a net loss in the momentum flux of the intermediate phase as well (mainly due to direct phase transition as seen in Figure 11), the amount of the momentum flux transferred from the intermediate phase to the warm phase is negligible compared to that from the hot phase. The warm phase gains about $50 \%$ of its original momentum flux, which is comparable to the loss from the hot phase. The momentum flux of the warm phase is dominated by the turbulent term, while the turbulent and thermal terms of the hot phase are similar (see Figure 6).

Lastly, Figure 13 plots the energy flux differences along with the cooling, SN energy injection rate, and rate of change of energy densities $\left(\langle\dot{E}\rangle_{\mathrm{ph}} / A\right)$, all normalized to $\left|\mathcal{F}_{E, \mathrm{w}}(z=1 \mathrm{kpc})\right|$. $\langle\dot{E}\rangle_{\mathrm{ph}}$ (dashed lines) is negligible for all phases, so energy balance is maintained between flux differences (solid lines) and source terms (dotted lines; cooling in warm and intermediate

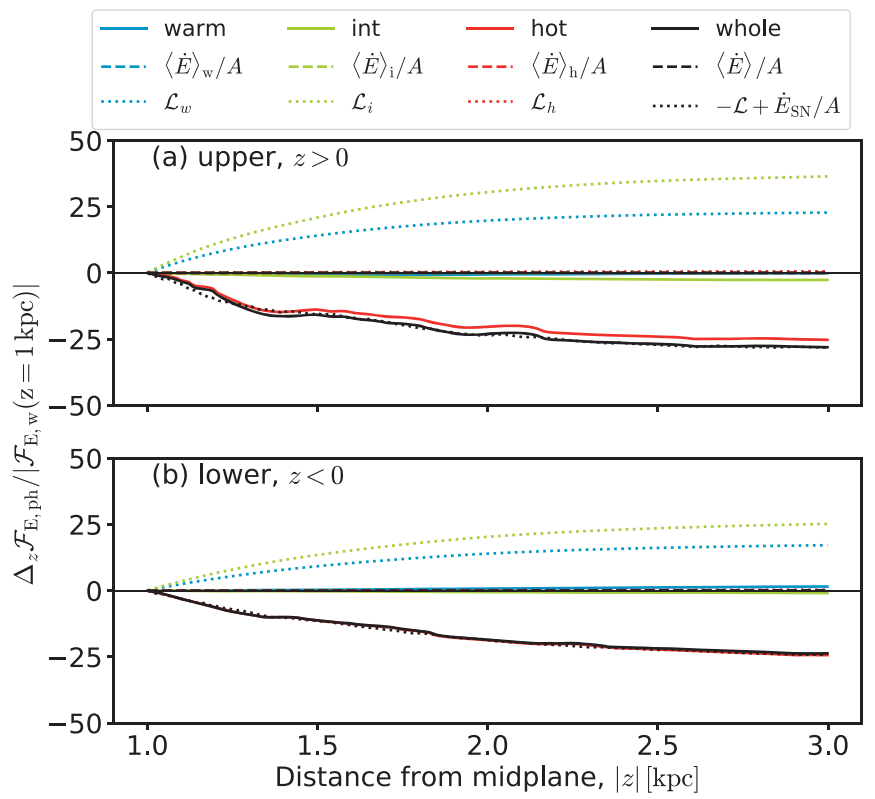

Figure 13. Same as Figure 11 , but for the energy flux difference $\left(\Delta_{z} \mathcal{F}_{\mathrm{E}, \mathrm{ph}}\right.$; Equation (18)). In addition, the net cooling rate per unit area of each thermal gas phase $\left(\mathcal{L}_{\mathrm{ph}}\right)$ is shown as dotted lines. The time-dependent energy changes in the volume $\langle\dot{E}\rangle_{\mathrm{ph}} / A$ (found by substituting $\rho \rightarrow \rho v^{2} / 2+P_{\mathrm{th}} /(\gamma-1)$ into Equation (16); dashed line) are negligible. The black dotted line denotes the total energy source term of the energy equation, Equation (15), including the net cooling and direct SN energy injection; this net loss (negative source) is an excellent match to the net energy flux change of the whole medium, most of which is loss of hot gas energy flux. Note that the range of the $y$-axis is about two orders of magnitude larger than that in Figures 11 and 12, demonstrating that a large amount of energy is brought into the extraplanar region via hotphase gas, but this is mostly lost to cooling.

phases, and direct $\mathrm{SN}$ energy injection from runaways). The hot gas energy flux drops with height $\left(\Delta_{z} \mathcal{F}_{E, \mathrm{~h}}<0\right.$; solid red line) but there is no significant increase of the energy flux in either the warm or intermediate phase. Instead, cooling losses from these two phases (dotted blue and green lines) are responsible for draining the hot gas energy, as well as for radiating away the additional $\mathrm{SN}$ energy injected within the volume. That is, $\dot{E}_{\mathrm{SN}} / A-\Delta_{z} \mathcal{F}_{E, \mathrm{~h}} \approx\left(\mathcal{L}_{\mathrm{w}}+\mathcal{L}_{\mathrm{i}}\right)$. The net loss of energy is large compared to the energy flux of the warm phase at $z_{i}$.

In summary, from Figures 11-13, we conclude that the warm phase gains mass flux from the intermediate phase and momentum flux from the hot phase. The energy flux available in the hot gas is enormous, but there are significant losses (20-30 times the original energy flux in the warm medium) over $\Delta z=2 \mathrm{kpc}$. Energy transfer between the hot and warm phases can occur by mixing or shocks, and the substantial amount of energy imparted by these routes is lost from the simulation rather than appearing in other phases, due to very efficient radiative cooling in both warm and intermediate phases.

Using the net mass and momentum exchanges, we can understand the ballistic model results presented in Section 3.2. The phase transition from the intermediate phase to the warm phase indeed occurs and is substantial in terms of mass flux. However, the momentum gain from the intermediate phase is insignificant, implying that the phase transition from the intermediate phase occurs preferentially at low velocities (or includes both outflowing and inflowing components). This is the reason why the ballistic approximation of the warm phase 

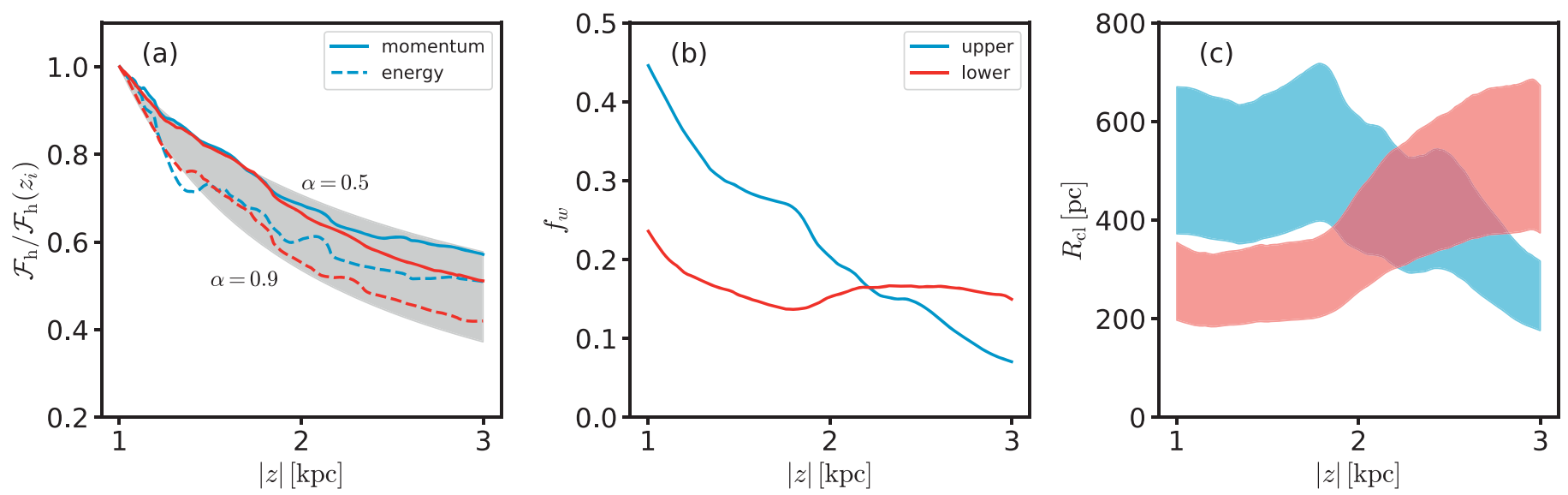

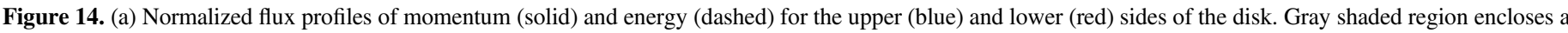

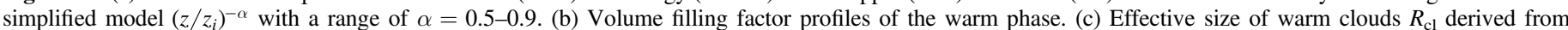

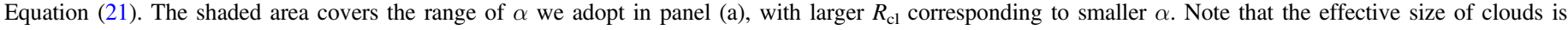
generally large, implying that in reality the warm phase is likely to interact with the hot winds as a single entity rather than many small cloudlets.

works relatively well even though there is non-negligible mass transfer from the intermediate phase. The excess of the highvelocity component in the warm phase seen in Figure 9 comes from the interaction with the hot phase, as demonstrated by the momentum flux changes in Figure 12. Overall, interaction between phases results in a factor of 1.5-2 increase in the mass and momentum fluxes of the warm phase over $\Delta z=2 \mathrm{kpc}$ compared to its steady-state injection fluxes. We note that the hot medium sees a 40\%-50\% reduction in both kinetic $\left(\rho v_{z}^{2}\right)$ and thermal $(P)$ momentum fluxes over the region studied.

\subsection{Effective Size of Warm Clouds}

Figure 12 shows that the net momentum flux gain of the warm medium with increasing $|z|$ (allowing for the reduction in momentum from the increase in $\Phi$ ) can be accounted for primarily by loss from the hot medium. The flux transfer could in principle be mediated by either mixing or shocks; with the present simulations, we cannot differentiate between these processes. ${ }^{13}$ In both cases, excess energy flux that is transferred from the hot medium to the warm and intermediate-temperature components in the interaction would be radiated away, as indeed is evident in Figure 13.

In this section, we use a simple interaction model to calculate the effective size of warm clouds based on the rate of flux loss from the hot medium. This allows us to distinguish whether the hot winds are interacting with many small cloudlets or a few big clouds.

We adopt a simple model in which (1) the warm gas is in the form of spherical clouds with radius $R_{\mathrm{cl}}$ and (2) the hot gas flux is "absorbed" by the warm clouds due to interaction. The flux loss of the hot gas can then be written as

$$
\frac{d \mathcal{F}_{h}}{d z}=-n_{\mathrm{cl}} A_{\mathrm{cl}} \mathcal{F}_{h}
$$

where $\mathcal{F}_{h}$ can either be the momentum or energy flux. Here, $A_{\mathrm{cl}}=\pi R_{\mathrm{cl}}^{2}$ is the cross section of the warm cloud, and $n_{\mathrm{cl}}=M_{\mathrm{w}} /\left(M_{\mathrm{cl}} V\right)$ is the number density of warm clouds, where $M_{\mathrm{w}}=\rho_{\mathrm{w}} V_{\mathrm{w}}$ is the total mass of warm gas and

\footnotetext{
13 The difference between the shock versus mixing-mediated transfer can be identified in idealized models where the hot gas is injected with a passive scalar; see Schneider et al. 2020).
}

$M_{\mathrm{cl}}=(4 / 3) \pi R_{\mathrm{cl}}^{3} \rho_{\mathrm{w}}$ is the mass of one cloud. We can rearrange Equation (20) as

$$
\frac{d \ln \mathcal{F}_{h}}{d z}=-\frac{3}{4} \frac{f_{w}}{R_{\mathrm{cl}}}
$$

where $f_{w}=V_{w} / V$ is the volume fraction of the warm phase. We note that the interaction (flux loss) is more efficient with a smaller cloud size as the effective cross section increases with many small clouds.

In Figure 14(a), we first plot the normalized momentum (solid) and energy (dashed) fluxes, $\mathcal{F}_{h} / \mathcal{F}_{h}\left(z_{i}\right)$, in the upper (blue) and lower (red) sides of the disk. We note that the energy and momentum flux losses on a given side are similar to each other, consistent with the simple physical interaction model. Because the numerically measured flux is not a smooth function, its numerical derivative (lhs of Equation (21)) gives very noisy results. For the sake of clarity of presentation, we simply adopt a functional form, $\mathcal{F}_{h} / \mathcal{F}_{h}\left(z_{i}\right)=\left(z / z_{i}\right)^{-\alpha}$, with a range of $\alpha$ from 0.5 to 0.9 that describes the general behavior of the flux loss (see the gray shaded region in Figure 14(a)). With the adopted power-law model, $d \ln \mathcal{F}_{h} / d z=-\alpha / z$.

Using the volume fraction of the warm phase measured directly from the simulation (Figure 14(b)), we obtain the cloud size $R_{\mathrm{cl}}=(3 / 4) f_{w} z / \alpha$ in Figure 14(c). Note that the larger $R_{\mathrm{cl}}$ corresponds to smaller $\alpha$ and less efficient interaction. The effective value of $R_{\mathrm{cl}}$ is generally large, $R_{\mathrm{cl}} \gtrsim$ a few $100 \mathrm{pc}$, implying that at high altitudes the warm phase that interacts with the hot winds exists more likely as one big entity rather than as small cloudlets. Indeed, the structure seen in Figure 1 is consistent with this; even though resolved small cloudlets are present, most of the warm medium is in fairly large structures. This results in a smaller effective cross section for the warmhot interaction than might be naively expected.

The interaction between warm clouds and hot, high-velocity flows has been extensively studied to understand whether hot winds can accelerate warm clouds before hydrodynamic instabilities break the clouds apart. Starting from hydrodynamic simulations of adiabatic shocks passing through spherical clouds (e.g., Klein et al. 1994; Xu \& Stone 1995), shockcloud interaction simulations have become ever more sophisticated, including, e.g., magnetic fields (e.g., Shin et al. 2008; McCourt et al. 2015), radiative cooling (e.g., Fragile et al. 2005; 
Cooper et al. 2009; Scannapieco \& Brüggen 2015), thermal conduction (e.g., Brüggen \& Scannapieco 2016), and turbulent clouds (e.g., Schneider \& Robertson 2017; Banda-Barragán et al. 2019). Among the physical processes considered, radiative cooling seems to prolong the survival of clouds, and, for a sufficiently large cloud size, the mixed gas promotes cooling to grow the total mass of the cool component (Marinacci et al. 2010; Armillotta et al. 2016; Gronke \& Oh 2018, 2020).

Gronke \& Oh (2018) have suggested that above a critical cloud size where $t_{\text {cool,mixed }}<t_{\mathrm{cc}}$, corresponding to

$$
\begin{gathered}
R_{\mathrm{cl}, \mathrm{crit}} \sim \frac{v_{\mathrm{wind}} t_{\text {cool,mixed }}}{\chi^{1 / 2}} \\
=109 \mathrm{pc}\left(\frac{v_{\text {wind }}}{100 \mathrm{~km} \mathrm{~s}^{-1}}\right)\left(\frac{t_{\text {cool,mixed }}}{10 \mathrm{Myr}}\right)\left(\frac{\chi}{100}\right)^{-1 / 2},
\end{gathered}
$$

clouds will grow in mass. In the above, we have normalized based on conditions in our simulations at $z \sim 1 \mathrm{kpc}$, where the density contrast between warm and hot is about $\chi \sim 100$, the relative velocity is $v_{\text {wind }} \sim 100 \mathrm{~km} \mathrm{~s}^{-1}$, and the cooling time in the intermediate phase is $t_{\text {cool, mix }} \sim 1-10 \mathrm{Myr}$.

Here, even though the effective warm cloud size we evaluate is quite large (Figure 14), we find that there is no significant mass added from the hot to the warm phase. This apparent discrepancy with the simple critical size estimation can be due to additional complexities that are missing in idealized shockcloud studies. In Gronke \& Oh (2018), the setup assumes hot, laminar winds with a large mass reservoir that is continuously blown, and cooling occurs in a wake behind the cloud. In our simulations, the hot reservoir is limited, and the bursty behavior of outflows leads to intermittent interactions between clouds and winds. Indeed, other simulations where the hot wind is turbulent, like our own, have not found evidence for interaction-triggered cooling of the hot medium, and have suggested that lateral turbulent flows limit the downstream condensation (Schneider et al. 2020).

\section{Summary and Discussion}

\subsection{Summary}

With the goal of improving understanding of multiphase outflows, in this paper we have developed and applied a method to investigate how different gas phases evolve and interact with each other during inflow/outflow cycles. Our application is to MHD simulations carried out using the TIGRESS numerical framework (Kim et al. 2017), which target star formation, ISM, and galactic potential conditions similar to the solar neighborhood. This simulation is ideal for the investigation of galactic outflows with complex interactions because (1) the simulation duration is quite long (nearly a gigayear), covering many star formation/feedback and outflow/inflow cycles; (2) star formation rates are self-regulated (hence self-consistent with the time-dependent multiphase ISM state) and $\mathrm{SNe}$ occur in star clusters and runaways, providing realistic spatiotemporal correlations of feedback sources with each other and with the surrounding ISM; and (3) the uniform spatial resolution employed in the simulation is necessary to correctly capture multiphase outflows and the interaction between them numerically. Kim \& Ostriker (2018) presented an initial analysis of this model to quantify the characteristics of the hot winds and warm fountains.
In this paper, we extend the previous analysis to compare with the predictions of a simple ballistic model and to quantify the exchange of mass, momentum, and energy flux among phases. To assess the extent of interactions quantitatively, we investigate the exchange of mass, momentum, and energy fluxes between thermal phases using the vertical profiles of the flux differences between the height of interest and launching (Figures 11-13). To isolate the exchange of fluxes between phases, it is important to factor out all other potential flux changes, including (1) the net mass change in the warm phase $\left(5050 \mathrm{~K}<T<2 \times 10^{4} \mathrm{~K}\right)$, considering that the steady-state assumption is imperfect, (2) the momentum loss as the gas climbs out of the gravitational potential, and (3) energy loss by cooling and gain by in situ SN explosions. After taking these additional "source" terms into account, we can directly link the excess/deficit of flux in one phase to the deficit/excess of flux in another phase.

Our main results are summarized as follows:

1. The predictions of the ballistic model approximate the kinematic distribution of the extraplanar warm gas in the simulation reasonably well at intermediate vertical velocities $\left(50 \mathrm{~km} \mathrm{~s}^{-1} \lesssim\left|v_{z}\right| \lesssim 100 \mathrm{~km} \mathrm{~s}^{-1}\right)$. The agreement between model and simulation data worsens at high velocities, where the ballistic model underestimates the amount of warm mass flux almost by a factor 2 (Figure 7). This result indicates that interaction with other phases may partially affect the warm gas kinematics.

2. Cooling of gas at intermediate temperatures transfers some mass flux from the intermediate phase $(2 \times$ $\left.10^{4} \mathrm{~K}<T<5 \times 10^{5} \mathrm{~K}\right)$ to the warm phase. From $|z|=1 \mathrm{kpc}$ to $|z|=3 \mathrm{kpc}$, the warm gas has gained almost $50 \%-100 \%$ of its initial mass flux from the intermediate phase (Figure 10,11). However, even when the v-PDFs of warm and intermediate gas are summed, a discrepancy between the ballistic model and the simulation results persists (Figure 9). The discrepancy is particularly large on the lower $(z<0)$ side of the disk.

3 . The missing piece is the exchange of momentum flux between warm and hot gas. The amount of momentum flux transferred from the hot to the warm phase within $|z|=3 \mathrm{kpc}$ is $50 \%$ of the momentum flux of the warm phase at the launching height, $z=1 \mathrm{kpc}$ (Figure 12). The warm gas gains considerable momentum flux from the hot gas, but negligible mass flux. This results in acceleration of the warm gas.

4. The energy flux of the hot phase shows huge losses between $|z|=1-3 \mathrm{kpc}$, but no other phase increases its energy flux. The energy flux lost from the hot gas is $\sim 20-30$ times the initial energy flux of the warm phase. The loss of energy flux from the hot medium (including the direct energy injection from $\mathrm{SNe}$ exploding in extraplanar regions) without a corresponding energy flux gain in another phase can be explained by strong cooling in both warm and intermediate phases.

5. Based on the flux changes in the hot phase and a simple interaction model with spherical clouds, we derive the effective cloud size of a few $100 \mathrm{pc}$ (Figure 14). The hot phase loses momentum and energy through interaction with a few large warm clouds rather than many small cloudlets. 


\subsection{Discussion}

We emphasize that a number of features particular to the TIGRESS simulation has enabled the quantitative analysis done in this paper. First, the simulation reaches a quasi-steady state in an average sense. Vertical dynamical equilibrium holds in the sense that the time-averaged momentum flux difference balances the weight of the gas (Figure 4(a)), while bursty star formation and inflow/outflow cycles are evident from the horizontally averaged spacetime diagram of mass fluxes (Figure 3). Covering several self-consistent star formation/ feedback cycles ( $\sim$ a few hundred Myr for solar neighborhood conditions) is important for studying fountains in detail; other local simulations have either run for much shorter duration (e.g., Gatto et al. 2017; Kannan et al. 2020) or adopted a prescribed feedback rate and positioning (e.g., Creasey et al. 2013; Girichidis et al. 2016; Martizzi et al. 2016; Li et al. 2017). As part of our analysis, we separate the gas into three different thermal phases and make use of horizontally and temporally averaged vertical profiles of physical quantities. Uniform resolution both near the midplane and in the extraplanar regions allows a fair phase separation within the simulated volume. In simulations with adaptive resolutions (using a semi-Lagrangian or adaptive mesh refinement code), typically the resolution is progressively poorer as flows move outward. Without sufficient resolution and phase separation at all altitudes, investigating interaction between phases in outflows is challenging, especially in the extraplanar region (e.g., Muratov et al. 2015; Hu 2019; Kannan et al. 2020). Currently, the only global simulations that employ uniform resolution at a similar level to that presented here is the CGOLS suite, in which a similar investigation of multiphase winds is underway (Schneider et al. 2020). However, star formation is not self-consistently modeled in the CGOLS suite; its focus is on winds in extreme starbursts rather than the mid-to-outer-disk regions of normal galaxies, where self-consistent modeling is essential for following the long-term outflow/inflow cycles of multiphase gas.

Although local models have unique advantages compared to global models, there are also caveats that must be borne in mind. With the periodic boundary conditions adopted in the horizontal directions, hot outflows can only expand to a limited extent as streamline separation cannot exceed the horizontal box size. This limits pressure gradients that would otherwise produce outward acceleration, while simultaneously limiting adiabatic cooling (e.g., Martizzi et al. 2016). Hot outflows in local models therefore would be slower and hotter than those in global models (Chevalier \& Clegg 1985). For outflows from the mid-disk regions under consideration, this global effect could require a box size of several tens of kiloparsecs (much larger than the box size required for the global expansion of a central starburst wind).

The limited horizontal domain is less of an issue for the warm fountain flow. Warm clouds fill only a small fraction of volume, and as their motions are already supersonic, streamline expansion would not produce acceleration of a warm outflow. In local simulations, the warm gas is able to rise up and fall back down while also moving horizontally (i.e., without "selfcollision"). Indeed, we find that horizontal velocity dispersions remain more or less constant in $z$ for the warm fountain flows; no sign of artificial collimation of flows is evident (if present, this would be seen as a decrease of lateral velocity dispersion in the vertical direction). Still, with a surrounding hot wind that may be somewhat slower than realistic (such that the momentum flux is lower for a given mass flux), the interaction between the warm and hot flows may differ quantitatively from the case where full global streamline expansion occurs. One way to explore this would be via controlled cloud-wind interaction simulations (with the cloud and wind at the same pressure), in which the hot wind has a given mass flux and Bernoulli parameter but varying Mach number; this would mimic interactions at a range of distances from the hot wind launch point.

The local model assumes that neighboring regions behave similarly, which is a reasonable assumption in mid-to-outer galactic disks. However, synchronization with neighbors can potentially affect feedback and outflow/inflow cycles. If the horizontal domain size is too small, superbubbles driven by clustered feedback fill up the entire volume near the midplane. In this case, the volume filling factor of the hot gas near the midplane would show extreme behavior, oscillating between 0 and 1, and synchronized vertical oscillations would be exaggerated. With these concerns in mind, during the model development stage, we experimented with the horizontal box size and found that $L_{x}=L_{y} \approx 1 \mathrm{kpc}$ is an optimal choice for solar neighborhood conditions to minimize artificial correlations while still keeping the computational cost low enough to achieve high resolution and long-term evolution. ${ }^{14}$ With a horizontal domain of $(1 \mathrm{kpc})^{2}$ that covers more than one region of coherent star formation and feedback, the hot gas volume filling factor within $|z|<H$ fluctuates between 0.2 and 0.5 (Kim et al. 2017). By the time $t>200 \mathrm{Myr}$, which is used for our analysis, vertical oscillations have become irregular (compared to the regular oscillations at $t<100$ Myr triggered by initial transients; see Figure 3). This also demonstrates why it is important to evolve simulations long enough to erase the memory from the initial conditions.

Another possible caveat that should be kept in mind is that the simulations analyzed here did not include thermal conduction, which might alter the interaction between different gas phases. The effect of thermal conduction is to transfer thermal energy from a hotter to a colder medium, potentially leading warm gas to evaporate in the surrounding hotter material (e.g., Cowie \& McKee 1977; Weaver et al. 1977; Brüggen \& Scannapieco 2016; Armillotta et al. 2017). However, whether an effective phase transition-with the resulting addition of mass to the intermediate/hot phaseoccurs depends on the balance between thermal conduction and radiative cooling. If the cooling time is smaller than the evaporation time, the newly generated intermediate gas will cool quickly, returning mass to the warm phase and radiating away the thermal energy initially transferred from the hot to the warm phase. In addition, interfaces where conduction occurs are likely also to have turbulent mixing. Even if conductively heated gas does not have a short cooling time, mixing with nearby dense gas may lead to efficient radiation of the energy conducted out of the hot medium. In general, it can be expected that conductive heating increases the mass of hot gas and therefore the potential mass loading of hot winds, while mixing that leads to cooling limits the energy loading of winds (e.g., El-Badry et al. 2019). We note however that the effect of thermal conduction might be mitigated by the presence of magnetic fields. The motion of the conducting electrons is

\footnotetext{
${ }^{14}$ We note that the optimal horizontal domain size for local simulations differs for different galactic conditions
} 
parallel to the magnetic field lines, so that the efficiency of thermal conduction is strongly reduced in the transverse direction (e.g., Braginskii 1965; Orlando et al. 2008).

The results presented in this work are highly relevant to the interpretation of extraplanar gas kinematics in Milky Way-like galaxies. Recently, there have been some attempts to model H I and $\mathrm{H} \alpha$ extraplanar gas in the Milky Way and nearby starforming galaxies by using ballistic models (Collins et al. 2002; Fraternali \& Binney 2006; Marasco et al. 2012). Although these models have been able to reproduce the extraplanar gas profiles, they are unable to reproduce its kinematics. Interaction with surrounding gas is required to explain the observations (Fraternali \& Binney 2008; Marasco et al. 2012), although the origin of this interaction has been poorly investigated.

Galaxy properties affect the gas properties at the launch location, which, in turn, drive the level of interaction during the outflow evolution. In the simulation studied here, for example, the mass and momentum fluxes in the warm medium are larger than other phases at launch, suggesting at lowest order that a ballistic fountain model may be appropriate for this low-fillingfactor component, ignoring interactions with other components.

In global simulations of a dwarf galaxy (virial mass of $10^{10} M_{\odot}$ with gas mass of $\left.10^{7} M_{\odot}\right)$, Hu (2019) instead finds that the interaction between hot and warm gas is very effective in accelerating warm gas to velocities larger than the galaxy escape velocity $\left(v_{\mathrm{esc}} \sim 100 \mathrm{~km} \mathrm{~s}^{-1}\right)$. This might be due to the combined effect of a weaker gravitational potential (lower galaxy escape velocity) and higher energy loading factors of the hot gas. A more quantitative understanding of the reason behind these differences could be obtained by applying the analysis methods presented here to global simulations.

Finally, we highlight that thorough study of the outflow properties in high-resolution local disk simulations, such as the one performed in this paper, can provide the detailed information required to build subgrid models for multiphase wind launching and evolution in cosmological galaxy formation simulations. Given their resolving power for both midplane and extraplanar regions, high-resolution local simulations have clear advantages for quantifying both outflow loading and evolution, which can then be applied in lowerresolution, larger-scale simulations and/or semianalytic models. Using local simulations with imposed $\mathrm{SN}$ rates and positions, Creasey et al. $(2013,2015)$ ran a large suite of local simulations with varying galactic conditions and used their results to provide scaling relations for mass and metal loading factors (but without phase separation). Their parameterization with respect to local galactic conditions has been adopted in the semianalytic, radially resolved subgrid model of Forbes et al. (2019). Also using local simulations with imposed SN rates and positions, Li et al. (2017) separately measured mass, energy, and metal loading factors for warm and hot outflows, and these have been adopted in idealized global simulations to study wind-CGM interactions ( $\mathrm{Li} \&$ Tonnesen 2019). Utilizing the TIGRESS framework, a suite of local simulations with fully self-consistent treatment of star formation and feedback at varying galactic conditions has been analyzed to provide mass, momentum, energy, and metal loading factors as well as outflow velocities as a function of local galactic properties (C.G. Kim et al. 2020, in preparation). Applying the analysis presented in this paper to all other TIGRESS models would be the next step toward development of a subgrid wind "evolution/interaction" model. We anticipate that both "launching" and "evolution" subgrid treatments derived from resolved simulations will be valuable for future cosmological modeling.

We thank the anonymous referee for valuable comments and suggestions. A.V. received travel support from ITS, SERB, Government of India, and would like to thank Biman B. Nath and Prateek Sharma for useful discussions and encouragement. The work of C.-G.K. was partly supported by a grant from the Simons Foundation (CCA 528307, E.C.O.). The work of E.C.O. and C.-G.K. was partly supported by NASA ATP grant NNX17AG26G. L.A. acknowledges support from the Australian Research Council's Discovery Projects and Future Fellowships funding schemes, awards DP190101258 and FT180100375. This work was initiated as a project for the Kavli Summer Program in Astrophysics held at the Center for Computational Astrophysics of the Flatiron Institute in 2018. The program was cofunded by the Kavli Foundation and the Simons Foundation, and we thank them for their generous support. Resources supporting this work were provided in part by the NASA High-End Computing (HEC) Program through the NASA Advanced Supercomputing (NAS) Division at Ames Research Center, in part by the Princeton Institute for Computational Science and Engineering (PICSciE) and the Office of Information Technology's High Performance Computing Center, and in part by the National Energy Research Scientific Computing Center, which is supported by the Office of Science of the U.S. Department of Energy under contract No. DE-AC02-05CH11231.

\section{ORCID iDs}

Aditi Vijayan (10 https://orcid.org/0000-0002-7714-2379 Chang-Goo Kim (iD https://orcid.org/0000-0003-2896-3725 Lucia Armillotta (1) https://orcid.org/0000-0002-5708-1927 Eve C. Ostriker (i) https://orcid.org/0000-0002-0509-9113 Miao Li (iD https://orcid.org/0000-0003-0773-582X

\section{References}

Anglés-Alcázar, D., Faucher-Giguère, C.-A., Kereš, D., et al. 2017, MNRAS, 470, 4698

Armillotta, L., Fraternali, F., \& Marinacci, F. 2016, MNRAS, 462, 4157

Armillotta, L., Fraternali, F., Werk, J. K., Prochaska, J. X., \& Marinacci, F. 2017, MNRAS, 470, 114

Banda-Barragán, W. E., Zertuche, F. J., Federrath, C., et al. 2019, MNRAS, 486, 4526

Bizyaev, D., Walterbos, R. A. M., Yoachim, P., et al. 2017, ApJ, 839, 87

Bolatto, A. D., Warren, S. R., Leroy, A. K., et al. 2013, Natur, 499, 450

Braginskii, S. I. 1965, RvPP, 1, 205

Bregman, J. N. 1980, ApJ, 236, 577

Brüggen, M., \& Scannapieco, E. 2016, ApJ, 822, 31

Chen, Y.-M., Tremonti, C. A., Heckman, T. M., et al. 2010, AJ, 140, 445

Chevalier, R. A., \& Clegg, A. W. 1985, Natur, 317, 44

Chisholm, J., Tremonti, C. A., Leitherer, C., \& Chen, Y. 2017, MNRAS, 469, 4831

Christensen, C. R., Davé, R., Governato, F., et al. 2016, ApJ, 824, 57

Collins, J. A., Benjamin, R. A., \& Rand, R. J. 2002, ApJ, 578, 98

Cooper, J. L., Bicknell, G. V., Sutherland, R. S., \& Bland-Hawthorn, J. 2009, ApJ, 703, 330

Cowie, L. L., \& McKee, C. F. 1977, ApJ, 211, 135

Creasey, P., Theuns, T., \& Bower, R. G. 2013, MNRAS, 429, 1922

Creasey, P., Theuns, T., \& Bower, R. G. 2015, MNRAS, 446, 2125

Davé, R., Thompson, R., \& Hopkins, P. F. 2016, MNRAS, 462, 3265

El-Badry, K., Ostriker, E. C., Kim, C.-G., Quataert, E., \& Weisz, D. R. 2019, MNRAS, 490, 1961

Emerick, A., Bryan, G. L., \& Mac Low, M.-M. 2019, MNRAS, 482, 1304

Fielding, D., Quataert, E., \& Martizzi, D. 2018, MNRAS, 481, 3325 
Forbes, J. C., Krumholz, M. R., \& Speagle, J. S. 2019, MNRAS, 487, 3581 Ford, H. A., Lockman, F. J., \& McClure-Griffiths, N. M. 2010, ApJ, 722, 367 Fragile, P. C., Anninos, P., Gustafson, K., \& Murray, S. D. 2005, ApJ, 619, 327

Fraternali, F., \& Binney, J. J. 2006, MNRAS, 366, 449

Fraternali, F., \& Binney, J. J. 2008, MNRAS, 386, 935

Fraternali, F., van Moorsel, G., Sancisi, R., \& Oosterloo, T. 2002, AJ, 123,3124

Gaensler, B. M., Madsen, G. J., Chatterjee, S., \& Mao, S. A. 2008, PASA, 25,184

Gatto, A., Walch, S., Naab, T., et al. 2017, MNRAS, 466, 1903

Girichidis, P., Walch, S., Naab, T., et al. 2016, MNRAS, 456, 3432

Gronke, M., \& Oh, S. P. 2018, MNRAS, 480, L111

Gronke, M., \& Oh, S. P. 2020, MNRAS, 492, 1970

Haffner, L. M., Reynolds, R. J., Tufte, S. L., et al. 2003, ApJS, 149, 405

Heckman, T. M., Alexandroff, R. M., Borthakur, S., Overzier, R., \& Leitherer, C. 2015, ApJ, 809, 147

Hopkins, P. F., Quataert, E., \& Murray, N. 2012, MNRAS, 421, 3522

Hopkins, P. F., Wetzel, A., Kereš, D., et al. 2018, MNRAS, 477, 1578

Hu, C.-Y. 2019, MNRAS, 483, 3363

Iffrig, O., \& Hennebelle, P. 2017, A\&A, 604, A70

Kannan, R., Marinacci, F., Simpson, C. M., Glover, S. C. O., \& Hernquist, L. 2020, MNRAS, 491, 2088

Kim, C.-G., \& Ostriker, E. C. 2015a, ApJ, 802, 99

Kim, C.-G., \& Ostriker, E. C. 2015b, ApJ, 815, 67

Kim, C.-G., \& Ostriker, E. C. 2017, ApJ, 846, 133

Kim, C.-G., \& Ostriker, E. C. 2018, ApJ, 853, 173

Kim, C.-G., Ostriker, E. C., \& Kim, W.-T. 2013, ApJ, 776, 1

Kim, C.-G., Ostriker, E. C., \& Raileanu, R. 2017, ApJ, 834, 25

Kimm, T., \& Cen, R. 2014, ApJ, 788, 121

Klein, R. I., McKee, C. F., \& Colella, P. 1994, ApJ, 420, 213

Kuijken, K., \& Gilmore, G. 1989, MNRAS, 239, 605

Leitherer, C., Schaerer, D., Goldader, J. D., et al. 1999, ApJS, 123, 3

Leroy, A. K., Walter, F., Martini, P., et al. 2015, ApJ, 814, 83

Levy, R. C., Bolatto, A. D., Sánchez, S. F., et al. 2019, ApJ, 882, 84

Li, M., Bryan, G. L., \& Ostriker, J. P. 2017, ApJ, 841, 101

Li, M., \& Tonnesen, S. 2019, arXiv:1910.14235

Lockman, F. J. 1984, ApJ, 283, 90

Lockman, F. J. 2002, ApJL, 580, L47

Marasco, A., \& Fraternali, F. 2011, A\&A, 525, A134

Marasco, A., Fraternali, F., \& Binney, J. J. 2012, MNRAS, 419, 1107

Marasco, A., Fraternali, F., Heald, G., et al. 2019, A\&A, 631, A50

Marinacci, F., Binney, J., Fraternali, F., et al. 2010, MNRAS, 404, 1464

Marinacci, F., Sales, L. V., Vogelsberger, M., Torrey, P., \& Springel, V. 2019, MNRAS, 489, 4233

Martin, C. L. 2005, ApJ, 621, 227

Martini, P., Leroy, A. K., Mangum, J. G., et al. 2018, ApJ, 856, 61
Martizzi, D., Fielding, D., Faucher-Giguère, C.-A., \& Quataert, E. 2016, MNRAS, 459, 2311

McCourt, M., O’Leary, R. M., Madigan, A.-M., \& Quataert, E. 2015, MNRAS, 449, 2

Muratov, A. L., Kereš, D., Faucher-Giguère, C.-A., et al. 2015, MNRAS, 454, 2691

Naab, T., \& Ostriker, J. P. 2017, ARA\&A, 55, 59

Orlando, S., Bocchino, F., Reale, F., Peres, G., \& Pagano, P. 2008, ApJ, 678,274

Peek, J. E. G., Heiles, C., Douglas, K. A., et al. 2011, ApJS, 194, 20

Pillepich, A., Springel, V., Nelson, D., et al. 2018, MNRAS, 473, 4077

Qu, Z., \& Bregman, J. N. 2019, ApJ, 880, 89

Reynolds, R. J. 1991, ApJL, 372, 17

Rosdahl, J., Schaye, J., Dubois, Y., Kimm, T., \& Teyssier, R. 2017, MNRAS, 466, 11

Scannapieco, E., \& Brüggen, M. 2015, ApJ, 805, 158

Schaye, J., Crain, R. A., Bower, R. G., et al. 2015, MNRAS, 446, 521

Schneider, E. E., Ostriker, E. C., Robertson, B. E., \& Thompson, T. A. 2020, arXiv:2002.10468

Schneider, E. E., \& Robertson, B. E. 2017, ApJ, 834, 144

Shapiro, P. R., \& Field, G. B. 1976, ApJ, 205, 762

Shin, M.-S., Stone, J. M., \& Snyder, G. F. 2008, ApJ, 680, 336

Smith, M. C., Sijacki, D., \& Shen, S. 2018, MNRAS, 478, 302

Somerville, R. S., \& Davé, R. 2015, ARA\&A, 53, 51

Sparre, M., Pfrommer, C., \& Vogelsberger, M. 2019, MNRAS, 482, 5401

Springel, V., \& Hernquist, L. 2003, MNRAS, 339, 312

Stone, J. M., \& Gardiner, T. 2009, NewA, 14, 139

Stone, J. M., \& Gardiner, T. A. 2010, ApJS, 189, 142

Stone, J. M., Gardiner, T. A., Teuben, P., Hawley, J. F., \& Simon, J. B. 2008, ApJS, 178, 137

Strickland, D. K., \& Heckman, T. M. 2007, ApJ, 658, 258

Strickland, D. K., Heckman, T. M., Colbert, E. J. M., Hoopes, C. G., \& Weaver, K. A. 2004, ApJ, 606, 829

Teng, S. H., Veilleux, S., \& Baker, A. J. 2013, ApJ, 765, 95

van Woerden, H., Wakker, B. P., Schwarz, U. J., \& de Boer, K. S. 2004, High Velocity Clouds (Dordrecht: Kluwer), 312

Vasiliev, E. O., Shchekinov, Y. A., \& Nath, B. B. 2019, MNRAS, 486, 3685

Vijayan, A., Sarkar, K. C., Nath, B. B., Sharma, P., \& Shchekinov, Y. 2018 MNRAS, 475, 5513

Vogelsberger, M., Genel, S., Sijacki, D., et al. 2013, MNRAS, 436, 3031

Vogelsberger, M., Genel, S., Springel, V., et al. 2014, Natur, 509, 177

Walter, F., Weiss, A., \& Scoville, N. 2002, ApJL, 580, L21

Weaver, R., McCray, R., Castor, J., Shapiro, P., \& Moore, R. 1977, ApJ, 218,377

Xu, J., \& Stone, J. M. 1995, ApJ, 454, 172

Zschaechner, L. K., Rand, R. J., Heald, G. H., Gentile, G., \& Kamphuis, P. 2011, ApJ, 740, 35 